\title{
Type II chiral affine Lie algebras and string actions in doubled space
}

\author{
Machiko Hatsuda, ${ }^{a, b}$ Kiyoshi Kamimura $^{a}$ and Warren Siegel ${ }^{c, 1}$ \\ ${ }^{a}$ Physics Division, Faculty of Medicine, Juntendo University, \\ Chiba 270-1695, Japan \\ ${ }^{b}$ KEK Theory Center, High Energy Accelerator Research Organization, \\ Tsukuba, Ibaraki 305-0801, Japan \\ ${ }^{c}$ C.N. Yang Institute for Theoretical Physics State University of New York, \\ Stony Brook, NY 11794-3840, U.S.A. \\ E-mail: mhatsuda@post.kek.jp, kamimura@ph.sci.toho-u.ac.jp, \\ siegel@insti.physics.sunysb.edu
}

ABSTRACT: We present affine Lie algebras generated by the supercovariant derivatives and the supersymmetry generators for the left and right moving modes in the doubled space. Chirality is manifest in our doubled space as well as the T-duality symmetry. We present gauge invariant bosonic and superstring actions preserving the two-dimensional diffeomorphism invariance and the $\kappa$-symmetry where doubled spacetime coordinates are chiral fields. The doubled space becomes the usual space by dimensional reduction constraints.

KeYwords: Supersymmetry and Duality, Superstrings and Heterotic Strings, Space-Time Symmetries, String Duality

ArXiv EPrint: 1507.03061

\footnotetext{
${ }^{1}$ http://insti.physics.sunysb.edu/ siegel/plan.html.
} 


\section{Contents}

1 Introduction 1

2 Chiral affine Lie algebras $\quad 3$

2.1 Derivative operators and one form currents 4

2.2 Affine Lie algebras and $B$ field 6

2.3 "Chirality" from doubling 8

$\begin{array}{lll}3 & \text { Bosonic string action in doubled space } & 10\end{array}$

$\begin{array}{lll}3.1 & \text { Doubled chiral Poincaré generators } & 11\end{array}$

$\begin{array}{lll}3.2 & \text { Dimensional reduction constraints } & 14\end{array}$

$\begin{array}{lll}3.3 & \text { Bosonic string action } & 16\end{array}$

$\begin{array}{ll}3.4 \text { Gauge fixing } & 19\end{array}$

4 Superstring action in doubled space $\quad 20$

$\begin{array}{lll}4.1 & \text { Doubled chiral super-Poincaré generators } & 20\end{array}$

$\begin{array}{lll}4.2 & \text { Superstring action } & 22\end{array}$

5 Conclusions $\quad 25$

$\begin{array}{lr}\text { A Composition formula } & 26\end{array}$

B Nonlinear realization of nondegenerate Poincaré groups $\quad 27$

\section{Introduction}

The low energy effective field theory of the string theory has the T-duality symmetry. The worldsheet origin of the T-duality is mixing of momenta and winding modes of a string. These string modes are generalized to the supercovariant derivatives for a superstring which satisfy the affine super-Lie algebra [1-4]. Doubling spacetime coordinates makes the Tduality manifest [5-11]. A manifestly T-duality formulation based on the affine Lie algebra in the doubled space was proposed in [9-11]. The affine Lie algebra determines the new Lie bracket or equivalently C-bracket which gives rise to the stringy modification of the general coordinate transformation. The generalized geometry proposed in $[12,13]$ is described by the Courant bracket which is reduced from the C-bracket by the dimensional reduction. The generalized geometry and the double field theory have been widely studied [14-23] and review articles are [24, 25].

Recently we have proposed a manifestly T-duality formulation for a type II superstring [26, 27] and the one with the Ramond-Ramond gauge fields [28]. For a type II supersymmetric extension of the manifestly T-duality formalism chiral separation of 
affine Lie algebras is an essential problem. We specify chirality as left and right moving modes in a two dimensional worldsheet. Chiral currents for a bosonic string in a nonabelian background are constructed by the Wess-Zumion-Witten model [29]. A group element $g=g\left(\sigma^{+}\right) g^{\prime}\left(\sigma^{-}\right)$is considered where $\sigma^{ \pm}$are the left and right moving twodimensional coordinates. The left moving current is constructed as the right-invariant current, $\partial_{+} \underline{g g^{-1}}=\partial_{+} g g^{-1}\left(\sigma^{+}\right)$, while the right moving current is constructed as the leftinvariant current, $\underline{g}^{-1} \partial_{-} \underline{g}=g^{\prime-1} \partial_{-} g^{\prime}\left(\sigma^{-}\right)$. However it is known that the local supersymmetry generator (supercovariant derivative) is obtained from the left-invariant current, while the global supersymmetry generator is obtained from the right-invariant current for supersymmetric theories. For a type II superstring theory both the supercovariant derivative and the supersymmetry generator must have both the left and right moving modes, not one for each. Although the chiral separation of the supercovariant derivative algebra for a superstring on the anti-de Sitter space is obtained on the constrained surface [30, 31], the chiral separation of currents for a superstring in nonabelian backgrounds is in general still difficult.

In our formulation we begin by two independent Lie groups $G$ and $G^{\prime}$ which are parameterized by $Z^{M}$ and $Z^{M^{\prime}}$. For a direct product of the groups $\mathrm{G} \times \mathrm{G}^{\prime}$ a group element satisfies $\underline{g}=g\left(Z^{M}\right) g^{\prime}\left(Z^{M^{\prime}}\right)=g^{\prime}\left(Z^{M^{\prime}}\right) g\left(Z^{M}\right)$. Therefore the left-invariant and the right-invariant one forms contain both the left and right moving modes as $g^{-1} d g=g^{-1} d g+g^{-1} d g^{\prime}=$ $J\left(Z^{M}\right)+J\left(Z^{M^{\prime}}\right)$ and $d g g^{-1}=d g g^{-1}+d g^{\prime} g^{\prime-1}=\tilde{J}\left(Z^{M}\right)+\tilde{J}\left(Z^{M^{\prime}}\right)$. This is similar to the nonabelian currents given by Tseytlin $[6,7]$. The chiral scalar action used in that paper does not preserve the two-dimensional diffeomorphism invariance [32]. In our formulation a chiral scalar action preserves the two-dimensional diffeomorphism invariance allowing the $\kappa$-symmetry for the superstring action. The doubled coordinates are chiral fields, $Z^{M}\left(\sigma^{+}\right)$ and $Z^{M^{\prime}}\left(\sigma^{-}\right)$. The stringy geometry is governed by the affine Lie algebras generated by the chiral supercovariant derivatives. The covariant derivatives are still manifestly chiral even after reducing into the usual space by dimensional reduction constraints, since the dimensional reduction constraints are given by the auxiliary symmetry generators.

In order to construct a doubled space there are two types of doubling of a group G:

1. Semidirect product, $\mathrm{G} \rightarrow \mathrm{G} \ltimes \mathrm{G}^{*}$ :

A Lie group $G$ is generated by $\mathfrak{g}$ corresponding to derivative operators which include momenta, while another group $G^{*}$ is generated by $\mathfrak{g}^{*}$ corresponding to one form currents which include winding modes. This is a conventional way of doubling discussed in for example $[12,13,33]$. It gives the following inhomogeneous algebra

$$
[\mathfrak{g}, \mathfrak{g}]=\mathfrak{g}, \quad\left[\mathfrak{g}, \mathfrak{g}^{*}\right]=\mathfrak{g}^{*}, \quad\left[\mathfrak{g}^{*}, \mathfrak{g}^{*}\right]=0 .
$$

2. Direct product, $\mathrm{G} \rightarrow \mathrm{G} \times \mathrm{G}^{\prime}$ :

$\mathrm{G}$ and $\mathrm{G}^{\prime}$ are independent copy and they are generated by $\mathfrak{g}$ and $\mathfrak{g}^{\prime}$ corresponding to the left and right moving modes respectively. They satisfy the following algebra

$$
[\mathfrak{g}, \mathfrak{g}]=\mathfrak{g}, \quad\left[\mathfrak{g}, \mathfrak{g}^{\prime}\right]=0, \quad\left[\mathfrak{g}^{\prime}, \mathfrak{g}^{\prime}\right]=\mathfrak{g}^{\prime} .
$$


It is straightforward to construct one form currents and derivative operators which satisfy (1.1). In terms of them we present a general construction of chiral currents satisfying (1.2). This construction requires that the Lie group must have a nondegenerate group metric and the Lie algebra must have grading by the canonical dimension.

The organization of the paper is the following: in the next section we present a general construction of chiral affine Lie algebras generated by the supercovariant derivative and the symmetry generator. We begin by a Lie algebra with a nondegenerate group metric $[36,37]$. It is necessary to include the Lorentz $S_{m n}$ and its nondegenerate partner $\Sigma^{m n}$ for construction of two independent affine Lie algebras generated by the covariant derivative, $P_{m}$, and the symmetry generator, $\tilde{P}_{m}$. The Lie algebra is graded by a dilatation operator which plays an important role in this construction. Generators of the chiral affine algebras include the $B$ field. For a flat background the $B$ field can be written in terms of the dilatation operator.

In section 3 we present chiral affine Poincaré algebras in the doubled space and concrete expression of generators. A set of dimensional reduction constraints are examined which reduce the doubled space into the usual space with preserving the local geometry. We present an gauge invariant action for a bosonic string in the doubled space. Then we demonstrate that the string action in the doubled space is reduced into the usual action. In section 4 we present chiral affine super-Poincaré algebras in the doubled space. Then an gauge invariant action for a type II superstring in the doubled space is given. The super-doubled space is also reduced into the usual space.

\section{Chiral affine Lie algebras}

In this section we present a general construction of two sets of affine Lie algebras generated by the covariant derivatives and the symmetry generators.

- Affine Lie algebras

$\begin{array}{cccccc} & & & \text { particle } & \rightarrow & \text { string } \\ & & & & \text { affine Lie algebra } \\ \text { Lie algebra } G_{I} & \nearrow & \text { covariant derivative } & \dot{\circ}_{I} & \rightarrow & \dot{\triangleright}_{I}(\sigma) \\ \text { symmetry generator } & \tilde{\nabla}_{I} & \rightarrow & \tilde{\triangleright}_{I}(\sigma)\end{array}$

Next we double the Lie algebra as a direct product:

- Doubled chiral affine Lie algebras

$$
\begin{array}{lllll} 
& & \text { left } & \text { right } \\
\text { Lie algebra } G_{I} \rightarrow G_{M}, G_{M^{\prime}} & \searrow & \text { covariant derivative } & \check{\triangleright}_{M}\left(\sigma^{+}\right) & \check{\triangleright}_{M^{\prime}}\left(\sigma^{-}\right) \\
\text {symmetry generator } & \tilde{\triangleright}_{M}\left(\sigma^{+}\right) & \tilde{\triangleright}_{M^{\prime}}\left(\sigma^{-}\right)
\end{array}
$$

The doubled coordinates manifest chirality as well as the T-duality symmetry.

In subsection 2.1 at first for a given Lie algebra we construct the left-invariant current $J$, the right-invariant current $\tilde{J}$, the particle covariant derivative $\stackrel{\circ}{\nabla}$, and the particle symmetry generator $\tilde{\nabla}$. The derivatives and the currents satisfy the case 1 algebra in (1.1). 
The canonical dimensions of operators are expressed by an eigenvalue matrix of the dilatation operator. In subsection 2.2 the general construction of affine Lie algebras for the string covariant derivative $\dot{\triangleright}$, and the string symmetry generator $\tilde{\triangleright}$. They are linear combinations of the particle derivatives, $\stackrel{\circ}{\nabla}, \tilde{\nabla}$, and the $\sigma$-components of the currents, $J_{1}, \tilde{J}_{1}$, with the $B$ field as coefficients. In subsection 2.3 the Lie algebra is doubled. This doubling corresponds to the case 2 algebra in (1.2). The way of the doubling gives chiral affine Lie algebras.

\subsection{Derivative operators and one form currents}

A Lie algebra is generated by $G_{I}$ with

$$
\left[G_{I}, G_{J}\right\}=i f_{I J}{ }^{K} G_{K}
$$

where $[A, B\}=A B-(-)^{A B} B A$ is the graded commutator. A nondegenerate group metric $\eta_{I J}$ is introduced in such a way that the structure constant $f_{I J}{ }^{K}$ with lowered indices becomes totally graded antisymmetric

$$
\operatorname{tr}\left(G_{I} G_{J}\right)=\eta_{I J}=\frac{1}{2} \eta_{(I J]}, \quad f_{I J K} \equiv f_{I J}{ }^{L} \eta_{L K}=\frac{1}{3 !} f_{[I J K)} .
$$

We introduce a dilatation operator $\hat{N}$ whose eigenvalues are canonical dimensions $n_{I}$ as

$$
\left[\hat{N}, G_{I}\right]=i N_{I}^{J} G_{J}=i n_{I} G_{I}
$$

The Jacobi identity of the dilatation operator, $\hat{N}$, and Lie algebra generators, $G_{I}$ 's , gives an identity

$$
\begin{aligned}
{\left[\hat{N},\left[G_{I}, G_{J}\right\}\right]+\left[G_{I},\left[G_{J}, \hat{N}\right]\right\}+(-)^{I J}\left[G_{J},\left[\hat{N}, G_{I}\right]\right\} } & =0 \\
\Rightarrow-N_{[J}{ }^{K} f_{I) K L}+f_{I J}{ }^{K} N_{K}{ }^{M} \eta_{M L}=\left(\tilde{n}_{L}-n_{I}-n_{J}\right) f_{I J L} & =0
\end{aligned}
$$

with

$$
\tilde{n}_{I} G_{I} \equiv \eta^{L J} N_{J}{ }^{K} \eta_{K I} G_{L}
$$

The sum of canonical dimensions of a nondegenerate pair is set to be $n_{0}$, and the sum of canonical dimensions of the lower indices of the structure constant in (2.4) becomes also $n_{0}$

$$
\begin{aligned}
\left(N \eta+\eta N^{T}\right)_{I J} & =\left(n_{I}+n_{J}\right) \eta_{I J}=n_{0} \eta_{I J} \\
\Rightarrow \tilde{n}_{I} & =\left(n_{0}-n_{I}\right), \quad\left(n_{0}-n_{K}-n_{I}-n_{J}\right) f_{I J K}=0 .
\end{aligned}
$$

We take $n_{0}=2$ in order to choose $n_{P}=1$ for $\eta_{P P}=1$.

Particle derivatives of the Lie algebra $\stackrel{\circ}{\nabla}_{I}$ and $\tilde{\nabla}_{I}$ are constructed at first. An element of the Lie group $g=g(Z)$ is parameterized by the coordinates $Z^{M}$. There are two kinds of currents which are invariant under right or left multiplicative actions: 
- The left-invariant current and the particle covariant derivative generating the right multiplication $g \rightarrow g g_{1}$ :

$$
\begin{aligned}
& J=g^{-1} d g=i J^{I} G_{I}, \quad d J=-J \wedge J, \quad J^{I}=d Z^{M} R_{M}{ }^{I} \\
& \stackrel{\circ}{\nabla}_{I}=\left(R^{-1}\right)_{I}{ }^{M} \frac{1}{i} \partial_{M}, \quad\left[\stackrel{\circ}{\nabla}_{I}, \stackrel{\circ}{\nabla}_{J}\right\}=-i f_{I J}{ }^{K} \stackrel{\circ}{\nabla}_{K} \\
& \Rightarrow \partial_{[M} R_{N)}^{K}=(-)^{N I} \frac{1}{2} R_{[M}^{I} R_{N)}^{J} f_{J I}^{K}
\end{aligned}
$$

- The right-invariant current (Noether current) and the particle symmetry generator generating the left multiplication $g \rightarrow g_{1} g$ :

$$
\begin{array}{crr}
\tilde{J}=(d g) g^{-1}=i \tilde{J}^{I} G_{I}, & d \tilde{J}=\tilde{J} \wedge \tilde{J}, & \tilde{J}^{I}=d Z^{M} L_{M}^{I} \\
\tilde{\nabla}_{I}=\left(L^{-1}\right)_{I}{ }^{M} \frac{1}{i} \partial_{M}, & {\left[\tilde{\nabla}_{I}, \tilde{\nabla}_{J}\right\}=i f_{I J}{ }^{K} \tilde{\nabla}_{K}} \\
\Rightarrow \partial_{[M} L_{N)}{ }^{K} & =-(-)^{N I} \frac{1}{2} L_{[M}{ }^{I} L_{N)}{ }^{J} f_{J I}{ }^{K} .
\end{array}
$$

- Independence of the right and left multiplications:

$$
\left[\stackrel{\circ}{\nabla}_{I}, \tilde{\nabla}_{J}\right\}=0 \rightarrow\left(\partial_{M} R_{N}^{I}\right) R^{-1}{ }_{I}^{L}=(-)^{N M}\left(\partial_{N} L_{M}{ }^{I}\right) L^{-1}{ }_{I}^{L}
$$

Particle derivatives are extended to the string affine algebra generators; $\stackrel{\circ}{\nabla}_{I} \rightarrow \stackrel{\circ}{\nabla}_{I}(\sigma)$ and $\tilde{\nabla}_{I} \rightarrow \tilde{\nabla}_{I}(\sigma) . \tau$ and $\sigma$ components of currents are denoted by $J^{I}=d \sigma^{i} J_{i}^{I}=d \tau J_{0}^{I}+$ $d \sigma J_{1}^{I}$ and $\tilde{J}^{I}=d \tau \tilde{J}_{0}^{I}+d \sigma \tilde{J}_{1}^{I}$. Currents $J_{1}^{I}$ and $\tilde{J}_{1}^{I}$ carry the canonical dimension $2-n_{I}$, since $\partial_{\sigma}$ carries the canonical dimension 2 where $\alpha^{\prime}$ is abbreviated. The indices of currents are lowered with $\eta_{I J}$ as $J_{I} \equiv J^{L} \eta_{L I}$ and $\tilde{J}_{I} \equiv \tilde{J}^{L} \eta_{L I}$, and they are covariant under $\stackrel{\circ}{\nabla}_{I}$ and $\tilde{\nabla}_{I}$ respectively. Derivatives and currents satisfy the case 1 . semidirect product $\mathrm{G} \ltimes \mathrm{G}^{*}$ in (1.1), where $\stackrel{\circ}{\nabla} \in \mathfrak{g}, J_{1}^{I} \in \mathfrak{g}^{*}$ and $\tilde{\nabla} \in \mathfrak{g}, \tilde{J}_{1}^{I} \in \mathfrak{g}^{*}$;

$$
\begin{aligned}
& \left\{\begin{array}{l}
{\left[\stackrel{\circ}{\nabla}_{I}(1), \stackrel{\circ}{\nabla}_{J}(2)\right\}=-i f_{I J}{ }^{K} \stackrel{\circ}{\nabla}_{K} \delta(2-1)} \\
{\left[\stackrel{\circ}{\nabla}_{I}(1), J_{1}^{J}(2)\right\}=-i f_{K I}^{J} J_{1}^{K} \delta(2-1)-i \delta_{I}^{J} \partial_{\sigma} \delta(2-1)} \\
{\left[J_{1}^{I}(1), J_{1}^{J}(2)\right\}=0}
\end{array}\right. \\
& \left\{\begin{array}{l}
{\left[\tilde{\nabla}_{I}(1), \tilde{\nabla}_{J}(2)\right\}=i f_{I J}{ }^{K} \tilde{\nabla}_{K} \delta(2-1)} \\
{\left[\tilde{\nabla}_{I}(1), \tilde{J}_{1}^{J}(2)\right\}=i f_{K I}{ }^{J} \tilde{J}_{1}^{K} \delta(2-1)+i \delta_{I}^{J} \partial_{\sigma} \delta(2-1)} \\
{\left[\tilde{J}_{1}^{I}(1), \tilde{J}_{1}^{J}(2)\right\}=0}
\end{array}\right. \\
& \left\{\begin{array}{l}
{\left[\stackrel{\circ}{\nabla}_{I}(1), \tilde{\nabla}_{J}(2)\right\}=0} \\
{\left[\tilde{\nabla}_{I}(1), J_{1}^{J}(2)\right\}=-i M_{I}^{J}(2) \partial_{\sigma} \delta(2-1)} \\
{\left[\stackrel{\circ}{I}_{I}(1), \tilde{J}_{1}^{J}(2)\right\}=-i\left(M^{-1}\right)_{I}^{J}(2) \partial_{\sigma} \delta(2-1)}
\end{array}\right.
\end{aligned}
$$


The $\sigma$ coordinates of the two-dimensional space, $\sigma_{1}$ and $\sigma_{2}$, are denoted by 1 and 2 . The commutator between $\stackrel{\circ}{\nabla}_{I}$ and $\tilde{J}^{I}$ and the one between $\tilde{\nabla}_{I}$ and $J^{I}$ include a matrix $M_{I}^{J}$ which is defined as

$$
M_{I}^{J}=\left(L^{-1}\right)_{I}^{M} R_{M}^{J}
$$

It relates the left-invariant and the right-invariant currents, and it also relates the covariant derivative and the symmetry generator as

$$
\tilde{J}^{I} M_{I}^{K}=J^{K}, \quad \tilde{\nabla}_{I}=M_{I}^{K} \stackrel{\circ}{\nabla}_{K} .
$$

From the relation between currents $\tilde{J}$ and $J$ in (2.7) and (2.8)

$$
g^{-1} \tilde{J} g=g^{-1}\left(i d Z^{M} L_{M}^{I} G_{I}\right) g=i d Z^{M} R_{M}^{I} G_{I}=J \Rightarrow g^{-1} G_{I} g=M_{I}^{J} G_{J},
$$

$M_{I}^{J}$ satisfies an orthonormal condition as

$$
\begin{aligned}
\eta_{I J} & =\operatorname{tr}\left(G_{I} G_{J}\right)=\operatorname{tr}\left(g^{-1} G_{I} g g^{-1} G_{J} g\right)=M_{I}{ }^{L} M_{J}{ }^{K} \operatorname{tr}\left(G_{L} G_{K}\right) \\
\Rightarrow \quad \eta_{I J} & =M_{I}{ }^{L}(-)^{K(L+K)} M_{J}{ }^{K} \eta_{L K}=M_{I}{ }^{L}\left(M^{T}\right)^{K}{ }_{J} \eta_{L K} .
\end{aligned}
$$

The super-transpose of a matrix is given as $\left(M^{T}\right)^{K}{ }_{J}=(-)^{K(J+K)} M_{J}{ }^{K}$. From now on the super-transpose is implicitly imposed. Eq. (2.14) together with (2.15) leads to

$$
M_{I}^{P} M_{J}^{Q} M_{K}^{R} f_{P Q R}=f_{I J K}, \quad J^{I} J^{J} \eta_{I J}=\tilde{J}^{I} \tilde{J}^{J} \eta_{I J}
$$

Relations (2.7), (2.8) and (2.9) lead to that $M_{I}^{J}$ is a function of $Z^{M}$ satisfying

$$
i \tilde{\nabla}_{K} M_{I}^{J}=f_{I K}{ }^{L} M_{L}^{J}, \quad i \stackrel{\circ}{\nabla}_{K} M_{I}^{J}=M_{I}^{L} f_{L K}{ }^{J} .
$$

\subsection{Affine Lie algebras and $B$ field}

We construct two independent sets of affine Lie algebras generated by the covariant derivative and by the symmetry generator.

- Covariant derivative $\dot{\triangleright}_{I}$ :

$$
\left[\stackrel{\circ}{\triangleright}_{I}(1), \stackrel{\triangleright}{\triangleright}_{J}(2)\right\}=-i f_{I J}{ }^{K} \stackrel{\triangleright}{\triangleright}_{K} \delta(2-1)-i \eta_{I J} \partial_{\sigma} \delta(2-1)
$$

- Symmetry generator $\tilde{\triangleright}_{I}$ :

$$
\left[\tilde{\triangleright}_{I}(1), \tilde{\triangleright}_{J}(2)\right\}=i f_{I J}{ }^{K} \tilde{\triangleright}_{K} \delta(2-1)+i \eta_{I J} \partial_{\sigma} \delta(2-1)
$$

- Commutativity of $\stackrel{\triangleright}{\triangleright}_{I}$ and $\tilde{\triangleright}_{I}$ :

$$
\left[\stackrel{\triangleright}{\triangleright}_{I}(1), \tilde{\triangleright}_{J}(2)\right\}=0 \text {. }
$$


Jacobi identities of these affine algebras (2.18) and (2.19) require the existence of the nondegenerate group metric $\eta_{I J}$ and the totally antisymmetricity of the structure constant $f_{I J K}$ in $(2.2)$.

Let us set the generators of the affine algebras (2.18) and (2.19) in terms of currents and derivatives in (2.7), (2.8), (2.10) and (2.11) as

$$
\stackrel{\circ}{\triangleright}_{I}=\stackrel{\circ}{\nabla}_{I}+J_{1}^{K} b_{K I}, \quad \tilde{\triangleright}_{I}=\tilde{\nabla}_{I}+\tilde{J}_{1}^{K} \tilde{b}_{K I} .
$$

The algebras (2.18), (2.19) and (2.20) give conditions on $b_{I J}$ and $\tilde{b}_{I J}$ : the symmetric parts of $b_{I J}$ and $\tilde{b}_{I J}$ are uniquely determined from the signature of the Schwinger terms, the terms including $\partial_{\sigma} \delta(2-1)$, of the affine Lie algebras (2.18) and (2.19),

$$
b_{(I J]}=\eta_{I J}=-\tilde{b}_{(I J]} .
$$

Vanishing the coefficient of $\partial_{\sigma} \delta(2-1)$ in $(2.20)$ leads to

$$
\tilde{b}_{K I}=-b_{J L} M_{I}^{J} M_{K}{ }^{L} .
$$

Vanishing the coefficient of $\delta(2-1)$ in (2.20) using with (2.16) leads to

$$
\begin{aligned}
i \stackrel{\circ}{\nabla}_{[J} b_{I) K}-i \stackrel{\circ}{\nabla}_{K} b_{I J}-\eta^{L M} b_{M[J} f_{I) L K}+f_{I J}{ }^{L} b_{K L} & =0 \\
i \tilde{\nabla}_{[J \mid} \tilde{b}_{K \mid I)}-i \tilde{\nabla}_{K} \tilde{b}_{J I}-\eta^{M L} \tilde{b}_{[J \mid L} f_{\mid I) M K}+f_{I J}{ }^{L} \tilde{b}_{L K} & =0 .
\end{aligned}
$$

A simple solution of $b_{I J}$ and $\tilde{b}_{I J}$ is obtained from the Jacobi identity for $N_{I}^{J}$ given in (2.4) as

$$
\begin{aligned}
b_{K I} & =\frac{1}{2} N_{I}{ }^{L} \eta_{L K}=\frac{1}{2}\left(\eta_{K I}+B_{K I}\right) \\
B_{I K} & =-(-)^{I K} B_{K I}=\frac{1}{2} N_{[K \mid}{ }^{L} \eta_{L \mid I)}=\frac{1}{2}\left(n_{K}-n_{I}\right) \eta_{I K} \\
\tilde{b}_{K I} & =-\frac{1}{2} N_{L}^{M} \eta_{M J} M_{I}^{J} M_{K}^{L}=-\frac{1}{2}\left(\eta_{K I}+M_{K}{ }^{L} M_{I}^{J} B_{J L}\right) .
\end{aligned}
$$

In this solution $B_{I J}$ is constant and $M_{I}^{J}$ depends on parameters. There is an ambiguity in solutions of $b_{I J}$ and $\tilde{b}_{I J}$, which can be interchanged. The generators of the affine algebras are given as the followings:

$$
\begin{aligned}
& \stackrel{\triangleright}{\triangleright}_{I}=\stackrel{\circ}{\nabla}_{I}+\frac{1}{2} J_{1}^{K} N_{I}{ }^{L} \eta_{L K}=\stackrel{\circ}{\nabla}_{I}+\frac{1}{2}\left(J_{1}^{K} \eta_{K I}+J_{1}^{K} B_{K I}\right) \\
& \tilde{\triangleright}_{I}=\tilde{\nabla}_{I}-\frac{1}{2} \tilde{J}_{1}^{K} M_{K}{ }^{L} N_{L}{ }^{M} \eta_{M J} M_{I}^{J}=\tilde{\nabla}_{I}+\frac{1}{2}\left(-\tilde{J}_{1}^{K} \eta_{K I}+J_{1}^{K} B_{K J} M_{I}^{J}\right) .
\end{aligned}
$$

The $B$ field in the Wess-Zumino term is written as

$$
\begin{aligned}
B & =\frac{1}{2} d Z^{M} \wedge d Z^{N} B_{M N}=J^{I} \wedge J^{J} b_{I J}=\tilde{J}^{I} \wedge \tilde{J}^{J} \tilde{b}_{I J} \\
B_{M N} & =\frac{1}{2} R_{M}{ }^{I} R_{N}{ }^{J} b_{[I J)}=\frac{1}{2} L_{M}{ }^{I} L_{N}{ }^{J} \tilde{b}_{[I J)} .
\end{aligned}
$$

The three form $H=d B$ is written from (2.24) as

$$
H=d B=\frac{1}{3 !} f_{I J K} J^{I} \wedge J^{J} \wedge J^{K}=\frac{1}{3 !} f_{I J K} \tilde{J}^{I} \wedge \tilde{J}^{J} \wedge \tilde{J}^{K},
$$

and it is closed

$$
d H=\frac{1}{2} f_{I J K} d J^{I} \wedge J^{J} \wedge J^{K}=\frac{1}{2} f_{I J K} f_{L M}^{I} J^{L} \wedge J^{M} \wedge J^{J} \wedge J^{K}=0 .
$$




\section{3 "Chirality" from doubling}

Now we double the algebra (2.1) together with the nondegenerate metric (2.2) in the second way of doubling in (1.2) $\mathrm{G} \times \mathrm{G}^{\prime}$. Indices are denoted by $\underline{M}={ }_{\left(M, M^{\prime}\right)}$ corresponding to the left and right sectors. The structure constant $f_{\underline{M N}} \underline{\underline{L}}$, the nondegenerate group metric, $\eta_{M N}$ are extended as follows. Another metric $\hat{\eta}_{\underline{M N}}$ is introduced to define the Virasoro operator.

$$
\begin{aligned}
\mathrm{G} & \rightarrow \mathrm{G} \times \mathrm{G}^{\prime} \\
I & \rightarrow \underline{M}={ }_{\left(M, M^{\prime}\right)} \\
f_{I J}{ }^{K} & \rightarrow f_{\underline{M N}}^{\underline{L}}=\left(f_{M N}{ }^{L}, f_{M^{\prime} N^{\prime}}{ }^{\prime}=-f_{M N}{ }^{L}\right) \\
\eta_{I J} & \rightarrow \eta_{\underline{M N}}=\left(\eta_{M N}, \eta_{M^{\prime} N^{\prime}}=-\eta_{M N}\right) \\
\hat{\eta}_{I J} & \rightarrow \underline{\hat{\eta}_{M N}}=\left(\hat{\eta}_{M N}=\eta_{M N}, \hat{\eta}_{M^{\prime} N^{\prime}}=\eta_{M N}\right)
\end{aligned}
$$

Then we double the affine Lie algebras generated by the covariant derivative (2.18) and the one by the symmetry generator (2.19). Doubled affine Lie algebras are given as below:

- Covariant derivative $\stackrel{\circ}{\perp}_{\underline{M}}$ :

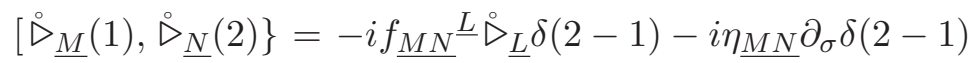

$$
\begin{aligned}
& \left\{\begin{array}{l}
{\left[\stackrel{\triangleright}{ }_{M}(1), \stackrel{\triangleright}{\triangleright}_{N}(2)\right\}=-i f_{M N}{ }^{L} \stackrel{\circ}{\triangleright}_{L} \delta(2-1)-i \eta_{M N} \partial_{\sigma} \delta(2-1)} \\
{\left[\stackrel{\circ}{\triangleright}_{M^{\prime}}(1), \stackrel{\triangleright}{\triangleright}_{N^{\prime}}(2)\right\}=i f_{M N}{ }^{L} \stackrel{\triangleright}{\triangleright}_{L^{\prime}} \delta(2-1)+i \eta_{M N} \partial_{\sigma} \delta(2-1)} \\
{\left[\stackrel{\circ}{\triangleright}_{M}(1), \stackrel{\circ}{\triangleright}_{N^{\prime}}(2)\right\}=0}
\end{array}\right.
\end{aligned}
$$

- Symmetry generator $\tilde{\nabla}_{\underline{M}}$ :

$$
\begin{gathered}
{\left[\tilde{\triangleright}_{\underline{M}}(1), \tilde{\triangleright}_{\underline{N}}(2)\right\}=i \underline{\underline{M N}}_{\underline{L}}^{\underline{L}} \tilde{\nabla}_{\underline{L}} \delta(2-1)+i \underline{\underline{M N}}_{\underline{N}} \partial_{\sigma} \delta(2-1)} \\
\left\{\begin{array}{l}
{\left[\tilde{\triangleright}_{M}(1), \tilde{\triangleright}_{N}(2)\right\}=i f_{M N} \tilde{\triangleright}_{L} \delta(2-1)+i \eta_{M N} \partial_{\sigma} \delta(2-1)} \\
{\left[\tilde{\triangleright}_{M^{\prime}}(1), \tilde{\triangleright}_{N^{\prime}}(2)\right\}=-i f_{M N}{ }^{L} \tilde{\triangleright}_{L^{\prime}} \delta(2-1)-i \eta_{M N} \partial_{\sigma} \delta(2-1)} \\
{\left[\tilde{\triangleright}_{M}(1), \tilde{\triangleright}_{N^{\prime}}(2)\right\}=0}
\end{array}\right.
\end{gathered}
$$

- The covariant derivative $\stackrel{\triangleright}{ }_{I}$ and the symmetry generator $\tilde{\triangleright}_{I}$ commute with each other,

$$
\left[\stackrel{\triangleright}{\triangleright}_{\underline{M}}(1), \tilde{\triangleright}_{\underline{N}}(2)\right\}=0
$$

The signature of the Schwinger term $\partial_{\sigma} \delta(2-1)$ in $(2.31)$ corresponds to the left or right chirality. 
Two-dimensional diffeomorphisms are generated by the Virasoro operators, $\mathcal{H}_{\tau}$ and $\mathcal{H}_{\sigma}$. The Virasoro operators and the Virasoro algebras are given by

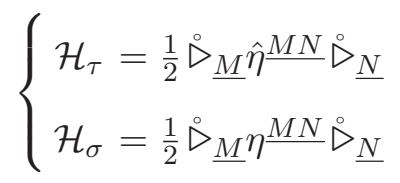

$$
\begin{aligned}
& {\left[\mathcal{H}_{\tau}(1), \mathcal{H}_{\tau}(2)\right]=i\left(\mathcal{H}_{\sigma}(1)+\mathcal{H}_{\sigma}(2)\right) \partial_{\sigma} \delta(2-1)} \\
& {\left[\mathcal{H}_{\sigma}(1), \mathcal{H}_{\tau}(2)\right]=i\left(\mathcal{H}_{\tau}(1)+\mathcal{H}_{\tau}(2)\right) \partial_{\sigma} \delta(2-1)} \\
& {\left[\mathcal{H}_{\sigma}(1), \mathcal{H}_{\sigma}(2)\right]=i\left(\mathcal{H}_{\sigma}(1)+\mathcal{H}_{\sigma}(2)\right) \partial_{\sigma} \delta(2-1) .}
\end{aligned}
$$

The two-dimensional diffeomorphisms of a function of double coordinates $\Phi\left(Z^{\underline{M}}\right)$ are given by

$$
\partial_{\tau} \Phi=i\left[\int d \sigma \mathcal{H}_{\tau}, \Phi\right], \quad \partial_{\sigma} \Phi=i\left[\int d \sigma \mathcal{H}_{\sigma}, \Phi\right] .
$$

The two dimensional left and right derivatives are given by

$$
\begin{aligned}
& \partial_{ \pm}=\frac{1}{2}\left(\partial_{\tau} \pm \partial_{\sigma}\right) \\
& \Rightarrow \quad \partial_{+} \Phi=i\left[\int d \sigma \mathcal{A}, \Phi\right], \quad \partial_{-} \Phi=i\left[\int d \sigma \mathcal{A}^{\prime}, \Phi\right] \\
& \left\{\begin{array}{l}
\mathcal{A}=\frac{1}{2}\left(\mathcal{H}_{\tau}+\mathcal{H}_{\sigma}\right)=\frac{1}{2} \stackrel{\circ}{\triangleright}_{M} \eta^{M N} \dot{\triangleright}_{N} \\
\mathcal{A}^{\prime}=\frac{1}{2}\left(\mathcal{H}_{\tau}-\mathcal{H}_{\sigma}\right)=\frac{1}{2} \stackrel{\triangleright}{\triangleright}_{M^{\prime}} \eta^{M^{\prime} N^{\prime} \stackrel{\triangleright}{\triangleright}_{N^{\prime}}}
\end{array}\right. \\
& {[\mathcal{A}(1), \mathcal{A}(2)]=i(\mathcal{A}(1)+\mathcal{A}(2)) \partial_{\sigma} \delta(2-1)} \\
& {\left[\mathcal{A}^{\prime}(1), \mathcal{A}^{\prime}(2)\right]=-i\left(\mathcal{A}^{\prime}(1)+\mathcal{A}^{\prime}(2)\right) \partial_{\sigma} \delta(2-1)} \\
& {\left[\mathcal{A}(1), \mathcal{A}^{\prime}(2)\right]=0}
\end{aligned}
$$

Therefore the covariant derivatives satisfy the left or right chiral conditions as

$$
\begin{aligned}
& \partial_{-} \Phi\left(Z^{M}\right)=0: \Phi\left(Z^{M}\right)=\left(\stackrel{\circ}{\nabla}_{M}, J^{M}, \stackrel{\circ}{\triangleright}_{M} ; \tilde{\nabla}_{M}, \tilde{J}^{M}, \tilde{\triangleright}_{M}\right) \\
& \partial_{+} \Phi\left(Z^{M^{\prime}}\right)=0: \Phi\left(Z^{M^{\prime}}\right)=\left(\stackrel{\circ}{\nabla}_{M^{\prime}}, J^{M^{\prime}},{\stackrel{\triangleright}{\boldsymbol{D}^{\prime}}}_{M^{\prime}} ; \tilde{\nabla}_{M^{\prime}}, \tilde{J}^{M^{\prime}}, \tilde{\nabla}_{M^{\prime}}\right)
\end{aligned}
$$

which comes from chiral property of the doubled coordinates $Z^{\underline{M}}$

$$
\partial_{-} Z^{M}=0=\partial_{+} Z^{M^{\prime}} \Rightarrow Z^{M}=Z^{M}\left(\sigma^{+}\right), Z^{M^{\prime}}=Z^{M^{\prime}}\left(\sigma^{-}\right) .
$$

For example the left-invariant current $J_{i}^{M}\left(\sigma^{+}\right)$are functions of only $\sigma^{+}$satisfying the Maurer-Cartan equation as

$$
\partial_{+} J_{-}^{M}-\partial_{-} J_{+}^{M}=J_{+}^{N} J_{-}^{K} f_{N K}{ }^{M} \Rightarrow \partial_{-} J_{+}^{M}\left(\sigma^{+}\right)=0 \text { and } J_{-}^{M}\left(\sigma^{+}\right)=0 .
$$

Similar relations hold for other currents. 
Let us consider the global $\mathrm{O}(\mathrm{n}, \mathrm{n})$ transformation where the number of the dimensions of the doubled space is $2 \mathrm{n}$ ( $\underline{M}$ runs 1 to $2 \mathrm{n}$ ). For a bosonic case $\mathrm{n}$ is given as sum of dimensions of $P_{m}, S_{m n}$ and $\Sigma^{m n}, \mathrm{n}=\mathrm{d}+2 \times \frac{\mathrm{d}(\mathrm{d}-1)}{2}=\mathrm{d}^{2}$ where $\mathrm{d}$ is the number of the momenta. Under the global $\mathrm{O}(\mathrm{n}, \mathrm{n}) \ni \mathcal{O}_{\underline{M}}^{\underline{L}}$ the covariant derivative (2.26) and (2.30) is transformed as

$$
{\stackrel{\triangleright}{\underline{M}^{M}}} \rightarrow \underline{\mathcal{O}}_{\underline{M}}^{\underline{L}} \stackrel{\circ}{\triangleright}_{\underline{L}}, \quad \mathcal{O}_{\underline{M}}^{\underline{L}} \eta_{\underline{L K}}\left(\mathcal{O}^{T}\right)^{\underline{K}}{ }_{\underline{N}}=\eta_{\underline{M N}} .
$$

Its affine Lie algebra (2.31) is transformed covariantly preserving the structure of $G \times G^{\prime}$ as

$$
f_{\underline{M N}}^{\underline{L}} \rightarrow \mathcal{O}_{\underline{M}}^{\underline{I}} \mathcal{O}_{\underline{M}}^{\underline{J}} f_{\underline{I J}}^{\underline{K}}\left(\mathcal{O}^{-1}\right)_{\underline{K}}^{\underline{L}} .
$$

Under the global $\mathrm{O}(\mathrm{n}, \mathrm{n})$ transformation the left and right moving modes are mixed and they are no more chiral operators in general. Under the $\mathrm{O}(\mathrm{n}, \mathrm{n})$ transformation the twodimensional $\sigma$-diffeomorphism constraint, $\mathcal{H}_{\sigma}$, is inert, but the $\tau$-diffeoporphism constraint, $\mathcal{H}_{\tau}$, causes the $\mathrm{O}(\mathrm{n}, \mathrm{n})$ transformation on the gravitational background fields $E_{\underline{A}} \underline{\underline{M}}$ as

$$
\mathcal{M}^{\underline{M N}}=\left(E^{T}\right)^{\underline{M}} \underline{A} \hat{\eta}^{\underline{A B}} E_{\underline{B}}^{\underline{N}} \rightarrow\left(\mathcal{O}^{T}\right)^{\underline{M}} \underline{L} \mathcal{M}^{\underline{L K}} \mathcal{O}_{\underline{K}} \underline{\underline{N}} .
$$

The $\mathrm{O}(\mathrm{n}, \mathrm{n})$ transformation is recognized as the coordinate transformation in the doubled space. An group element $\underline{g}\left(Z^{\underline{M}}\right)=g\left(Z^{M}\right) g^{\prime}\left(Z^{M^{\prime}}\right)=g^{\prime}\left(Z^{M^{\prime}}\right) g\left(Z^{M}\right)$ is transformed under the right multiplicative action of the $\mathrm{O}(\mathrm{n}, \mathrm{n})$ as

$$
\begin{aligned}
\underline{g}\left(Z^{\underline{M}}\right) & \rightarrow \underline{g}(\check{Z} \underline{M})=\underline{g}\left(Z^{\underline{M}}\right) \check{\mathcal{O}}=\underline{g}\left(Z^{\underline{M}}\right)+\delta \underline{g}\left(Z^{\underline{M}}\right) \\
Z^{\underline{M}} & \rightarrow \check{Z} \underline{M}=Z^{\underline{\underline{N}}} \mathcal{O}_{\underline{N}}^{\underline{M}} .
\end{aligned}
$$

Under the right multiplicative action which is denoted as

$$
\Delta=\underline{g}^{-1} \delta \underline{g}=(\check{\mathcal{O}}-1),
$$

the left-invariant one form $\underline{J}=\underline{g}^{-1} d \underline{g}$ and the right-invariant one form $\underline{\tilde{J}}=d \underline{g g}^{-1}$ are transformed as

$$
\delta \underline{J}=d \Delta+[\underline{J}, \Delta], \quad \delta \underline{\tilde{J}}=\underline{g}(d \Delta) \underline{g}^{-1} .
$$

Then the right-invariant one form is inert under the global $\mathrm{O}(\mathrm{n}, \mathrm{n})$ transformation, $d \Delta=$ $0 \Rightarrow \delta \underline{\tilde{J}}=0$. Hence the symmetry generator is inert under the global $\mathrm{O}(\mathrm{n}, \mathrm{n})$ transformation which rotates the covariant derivative.

\section{Bosonic string action in doubled space}

We begin with the nondegenerate Poincaré algebra including both the Lorentz generator and its nondegenerate partner $[30,31,37]$. Then it is doubled to construct chiral affine Poincaré algebras [26-28, 35, 36]. Concrete expression of the covariant derivatives and the symmetry generators for the left and right moving modes are given. The dimensional reduction constraints are examined on its consistency, chirality and the $\mathrm{O}(\mathrm{n}, \mathrm{n})$ transformation. A gauge invariant bosonic string action is presented, and dimensional reduction of the doubled space action is demonstrated. 


\subsection{Doubled chiral Poincaré generators}

Generators of nondegenerate Poincaré algebra are given by $G_{I}=\left(s_{m n}, p_{m}, \sigma^{m n}\right)$ with canonical dimensions $(0,1,2)$ respectively. The algebra is given as

$$
\begin{array}{rlrl}
\operatorname{dim} 0: & & {\left[s_{m n}, s_{l k}\right]} & =-i \eta_{[k \mid[m} s_{n] \mid l]} \\
\operatorname{dim} 1: & {\left[s_{m n}, p_{l}\right]} & =-i p_{[m} \eta_{n] l} \\
\operatorname{dim} 2: & {\left[s_{m n}, \sigma^{l k}\right]} & =-i \delta_{[m}^{[k} \sigma_{n]}^{l]},\left[p_{m}, p_{n}\right]=i \sigma_{m n} .
\end{array}
$$

The nondegenerate group metric is

$$
\eta_{I J}=\begin{array}{ccc}
s \\
p \\
\sigma
\end{array}\left(\begin{array}{lll} 
& p & \sigma \\
& & 1 \\
& 1 & \\
1 & &
\end{array}\right) .
$$

The nondegenerate Poincaré algebra (3.1) is extended to double affine Lie algebras. The covariant derivatives and the symmetry generators with the constant $b_{I J}$ solution in $(2.25)$ are given as follows:

- Covariant derivatives:

Flat left

$$
\begin{aligned}
& \stackrel{\triangleright}{M}_{M}=\left(S_{m n}, P_{m}, \Sigma^{m n}\right) \quad \triangleright_{M^{\prime}}=\left(S_{m^{\prime} n^{\prime}}, P_{m^{\prime}}, \Sigma^{m^{\prime} n^{\prime}}\right) \\
& \left\{\begin{array} { r l } 
{ S _ { m n } } & { = \stackrel { \circ } { \nabla } _ { S } } \\
{ P _ { m } } & { = \stackrel { \circ } { \nabla } _ { P } + \frac { 1 } { 2 } J _ { 1 } ^ { P } } \\
{ \Sigma ^ { m n } } & { = \stackrel { \circ } { \nabla } _ { \Sigma } + J _ { 1 } ^ { S } }
\end{array} \quad \left\{\begin{array}{rl}
S_{m^{\prime} n^{\prime}} & =\stackrel{\circ}{\nabla}_{S^{\prime}} \\
P_{m^{\prime}} & =\stackrel{\circ}{\nabla}_{P^{\prime}}-\frac{1}{2} J_{1}^{P^{\prime}} \\
\Sigma^{m^{\prime} n^{\prime}} & =\circ^{\circ} \\
\Sigma^{\prime} & -J_{1}^{S^{\prime}}
\end{array}\right.\right.
\end{aligned}
$$

- Symmetry generators:

Flat left $\tilde{\triangleright}_{M}=\left(\tilde{S}_{m n}, \tilde{P}_{m}, \tilde{\Sigma}^{m n}\right)$

$$
\begin{aligned}
& \left\{\begin{array}{l}
\tilde{S}_{m n}=\tilde{\nabla}_{S}-\left(\tilde{J}_{1}^{\Sigma}+\sum_{N=S, P} c_{S}^{N} \tilde{J}_{1}^{N}\right) \\
\tilde{P}_{m}=\tilde{\nabla}_{P}-\frac{1}{2}\left(\tilde{J}_{1}^{P}+\sum_{N=S} c_{P}^{N} \tilde{J}_{1}^{N}\right) \\
\tilde{\Sigma}^{m n}=\tilde{\nabla}_{\Sigma}
\end{array}\right. \\
& \text { Flat right } \tilde{\nabla}_{M^{\prime}}=\left(\tilde{S}_{m^{\prime} n^{\prime}}, \tilde{P}_{m^{\prime}}, \tilde{\Sigma}^{m^{\prime} n^{\prime}}\right) \\
& \left\{\begin{array}{l}
\tilde{S}_{m^{\prime} n^{\prime}}=\tilde{\nabla}_{S^{\prime}}+\left(\tilde{J}_{1}^{\Sigma^{\prime}}+\sum_{N^{\prime}=S^{\prime}, P^{\prime}} c_{S^{\prime}}^{N^{\prime}} \tilde{J}_{1}^{N^{\prime}}\right) \\
\tilde{P}_{m^{\prime}}=\tilde{\nabla}_{P^{\prime}}+\frac{1}{2}\left(\tilde{J}_{1}^{P^{\prime}}+\sum_{N^{\prime}=S^{\prime}} c_{P^{\prime}}^{N^{\prime}} \tilde{J}_{1}^{N^{\prime}}\right) \\
\tilde{\Sigma}^{m^{\prime} n^{\prime}}=\tilde{\nabla}_{\Sigma^{\prime}}
\end{array}\right.
\end{aligned}
$$


Symmetry generators include coefficients $c_{M}^{N}$ which are given from (2.23) and (2.24) as

$$
\sum_{N}^{n_{N}+n_{M}<2} c_{M}^{N} \tilde{J}_{1}^{N}=J_{1}^{N} B_{N L} M_{M}{ }^{L}, \quad \sum_{N^{\prime}}^{n_{N^{\prime}}+n_{M^{\prime}}<2} c_{M^{\prime}}^{N^{\prime}} \tilde{J}_{1}^{N^{\prime}}=-J_{1}^{N^{\prime}} B_{N^{\prime} L^{\prime}} M_{M^{\prime}}{ }^{L^{\prime}} .
$$

Nondegeneracy of the algebra requires only one chirality for flat left covariant derivative in (3.3) as $\stackrel{\circ}{\nabla}_{S}$ and $\stackrel{\circ}{\nabla}_{\Sigma}+J_{1}^{S}$, not both $\stackrel{\circ}{\nabla}_{S}$ and $\stackrel{\circ}{\nabla}_{\Sigma} \pm J_{1}^{S}$. Therefore (3.3) and (3.4) are unique representation of the affine nondegenerate algebras (2.18) and (2.19) up to the rescaling of currents.

Rescaled currents with parameters $\alpha$ and $\beta$ satisfy the following algebra modified from (2.18)

$$
\left[\triangleright_{I}(1), \triangleright_{J}(2)\right]=-i f_{I J}^{K} \stackrel{\triangleright}{\triangleright}_{K} \delta(2-1)-i \alpha \eta_{I J} \partial_{\sigma} \delta(2-1)
$$

as

$$
\left\{\begin{array}{rl}
S_{m n} & =\stackrel{\circ}{\nabla}_{S} \\
P_{m} & =\beta^{-1 / 2} \stackrel{\circ}{\nabla}_{P}+\frac{1}{2} \alpha \beta^{1 / 2} J_{1}^{P} \\
\Sigma^{m n} & =\beta^{-1} \stackrel{\circ}{\nabla}_{\Sigma}+\alpha \beta J_{1}^{S}
\end{array},\right.
$$

and analogous rescaling for another sector. The usual notation of the left and right moving modes is $P_{m}=\stackrel{\circ}{\nabla}_{P}+J_{1}^{P}$ for $\alpha=2, \beta=1$, or $P_{m}=\frac{1}{\sqrt{2}}\left(\stackrel{\circ}{\nabla}_{P}+J_{1}^{P}\right)$ for $\alpha=1, \beta=2$.

The Virasoro operators for a bosonic string in a flat space, $\mathcal{H}_{\tau}$ and $\mathcal{H}_{\sigma}$ are given by

$$
\begin{aligned}
\mathcal{H}_{\tau} & =\frac{1}{2} \stackrel{\triangleright}{\unrhd}_{\underline{M}} \hat{\eta}^{\underline{M N}}{\stackrel{\triangleright}{D_{N}}} \\
& =\frac{1}{2} P^{m} P_{m}+\frac{1}{2} P^{m^{\prime}} P_{m^{\prime}}+\frac{1}{2} \Sigma^{m n} S_{m n}+\frac{1}{2} \Sigma^{m^{\prime} n^{\prime}} S_{m^{\prime} n^{\prime}} \\
\mathcal{H}_{\sigma} & =\frac{1}{2} \stackrel{\triangleright}{\triangleright}_{\underline{M}} \eta^{\underline{M N}} \stackrel{\triangleright}{\triangleright}_{\underline{N}} \\
& =\frac{1}{2} P^{m} P_{m}-\frac{1}{2} P^{m^{\prime}} P_{m^{\prime}}+\frac{1}{2} \Sigma^{m n} S_{m n}-\frac{1}{2} \Sigma^{m^{\prime} n^{\prime}} S_{m^{\prime} n^{\prime}} .
\end{aligned}
$$

They satisfy the Virasoro algebra in (2.35). The two-dimensional chirality is determined by the Virasoro operators (2.40). Then a group element for the nondegenerate Poincaré algebra in (3.1) is parameterized as

$$
\begin{aligned}
& g=g\left(Z^{\underline{M}}\right)=g\left(Z^{M}\left(\sigma^{+}\right)\right) g\left(Z^{M^{\prime}}\left(\sigma^{-}\right)\right)
\end{aligned}
$$

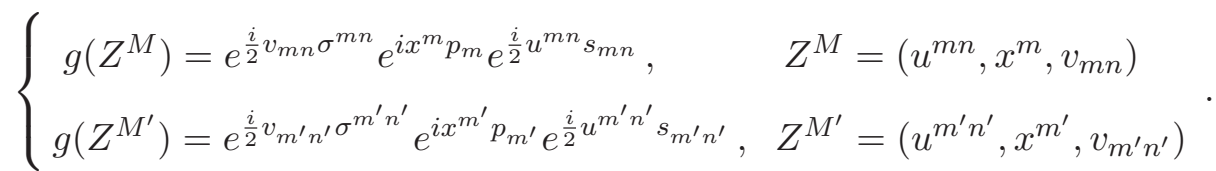

The $S_{m n}$ component of the left-invariant current is given by $g^{-1} d g=e^{-i u \cdot s} d e^{i u \cdot s}+$ $e^{-i u^{\prime} \cdot s^{\prime}} d e^{i u^{\prime} \cdot s^{\prime}}$ with $u \cdot s=\frac{1}{2} u^{m n} s_{m n}$;

$$
e^{-i u \cdot s} d e^{i u \cdot s}=\frac{e^{\operatorname{adj}_{u}}-1}{\operatorname{adj}_{u}} i d u \cdot s \equiv \Xi_{u}^{-1} i d u \cdot s, \quad \Xi_{u}=\frac{\operatorname{adj}_{u}}{e^{\operatorname{adj}_{u}-1}} .
$$

Details are in the appendix A. 
Concrete expression of the left-invariant current $J \underline{M}$ and the right-invariant current $\tilde{J} \underline{M}$ are obtained as follows:

- Left-invariant currents

$$
\begin{aligned}
& \text { Flat left } J^{M}=\left(J^{S}, J^{P}, J^{\Sigma}\right) \quad \\
& \left\{\begin{array} { l } 
{ J ^ { S } = e ^ { - u } d e ^ { u } = \Xi _ { u } ^ { - 1 } d u } \\
{ J ^ { P } = e ^ { - u } d x } \\
{ J ^ { \Sigma } = e ^ { - u } ( d v + \frac { 1 } { 2 } [ x , d x ] ) e ^ { u } }
\end{array} \quad \left\{\begin{array}{l}
J^{S^{\prime}}=-e^{u^{\prime}} d e^{-u^{\prime}} \\
J^{P^{\prime}}=e^{u^{\prime}} d x^{\prime} \\
J^{\Sigma^{\prime}}=e^{u^{\prime}}\left(d v^{\prime}-\frac{1}{2}\left[x^{\prime}, d x^{\prime}\right]\right) e^{-u^{\prime}}
\end{array}\right.\right.
\end{aligned}
$$

- Right-invariant currents

$$
\begin{array}{ll}
\text { Flat left } \tilde{J}^{M}=\left(\tilde{J}^{S}, \tilde{J}^{P}, \tilde{J}^{\Sigma}\right) \quad & : \text { Flat right } J^{M^{\prime}}=\left(J^{S^{\prime}}, J^{P^{\prime}}, J^{\Sigma^{\prime}}\right) \\
\left\{\begin{array} { l } 
{ \tilde { J } ^ { S } = - e ^ { u } d e ^ { - u } } \\
{ \tilde { J } ^ { P } = d x - \tilde { J } ^ { S } x } \\
{ \tilde { J } ^ { \Sigma } = d v - \frac { 1 } { 2 } [ x , \tilde { J } ^ { P } ] + [ v , \tilde { J } ^ { S } ] }
\end{array} \quad \left\{\begin{array}{l}
\tilde{J}^{S^{\prime}}=e^{-u^{\prime}} d e^{u^{\prime}} \\
\tilde{J}^{P^{\prime}}=d x^{\prime}+\tilde{J}^{S^{\prime}} x^{\prime} \\
\tilde{J}^{\Sigma^{\prime}}=d v^{\prime}+\frac{1}{2}\left[x^{\prime}, \tilde{J}^{P^{\prime}}\right]+\left[v^{\prime}, \tilde{J}^{S^{\prime}}\right]
\end{array}\right.\right.
\end{array}
$$

Detailed computation is given in the appendix B. From the above expression it is obvious that there is no difference bewteen $J^{P}$ and $\tilde{J}^{P}$ if $u$ is absent.

The covariant derivatives $\stackrel{\circ}{\nabla}_{\underline{M}}$ and the symmetry generators $\tilde{\nabla}_{\underline{M}}$ are obtained as follows.

- Covariant derivatives

$$
\begin{aligned}
& \text { Flat left } \stackrel{\circ}{\nabla}_{M}=\left(\stackrel{\circ}{\nabla}_{S}, \stackrel{\circ}{\nabla}_{P}, \stackrel{\circ}{\nabla}_{\Sigma}\right) \text { : Flat right } \stackrel{\circ}{\nabla}_{M^{\prime}}=\left(\stackrel{\circ}{\nabla}_{S^{\prime}}, \stackrel{\circ}{\nabla}_{P^{\prime}}, \stackrel{\circ}{\nabla}_{\Sigma^{\prime}}\right) \\
& \left\{\begin{array} { l } 
{ \stackrel { \circ } { \nabla } _ { S } = \Xi _ { u } \frac { 1 } { i } \partial _ { u } } \\
{ \stackrel { \circ } { \nabla } _ { P } = ( \frac { 1 } { i } \partial _ { x } - \frac { 1 } { 2 } x \frac { 1 } { i } \partial _ { v } ) e ^ { u } } \\
{ \stackrel { \circ } { \nabla } _ { \Sigma } = e ^ { - u } \frac { 1 } { i } \partial _ { v } e ^ { u } }
\end{array} \quad \left\{\begin{array}{l}
\stackrel{\circ}{\nabla}_{S^{\prime}}=\Xi_{-u^{\prime}} \frac{1}{i} \partial_{u^{\prime}} \\
\stackrel{\circ}{\nabla}_{P^{\prime}}=\left(\frac{1}{i} \partial_{x^{\prime}}+\frac{1}{2} x^{\prime} \frac{1}{i} \partial_{v^{\prime}}\right) e^{-u^{\prime}} \\
\stackrel{\circ}{\nabla}_{\Sigma^{\prime}}=e^{u^{\prime}} \frac{1}{i} \partial_{v^{\prime}} e^{-u^{\prime}}
\end{array}\right.\right.
\end{aligned}
$$

- Symmetry generators

$$
\begin{aligned}
& \text { Flat left } \tilde{\nabla}_{M}=\left(\tilde{\nabla}_{S}, \tilde{\nabla}_{P}, \tilde{\nabla}_{\Sigma}\right) \quad: \text { Flat right } \tilde{\nabla}_{M^{\prime}}=\left(\tilde{\nabla}_{S^{\prime}}, \tilde{\nabla}_{P^{\prime}}, \tilde{\nabla}_{\Sigma^{\prime}}\right) \\
& \left\{\begin{array} { l } 
{ \tilde { \nabla } _ { S } = \Xi _ { - u } \frac { 1 } { i } \partial _ { u } + [ x , \frac { 1 } { i } \partial _ { x } ] + [ v , \frac { 1 } { i } \partial _ { v } ] } \\
{ \tilde { \nabla } _ { P } = \frac { 1 } { i } \partial _ { x } + \frac { 1 } { 2 } x \frac { 1 } { i } \partial _ { v } } \\
{ \tilde { \nabla } _ { \Sigma } = \frac { 1 } { i } \partial _ { v } }
\end{array} \quad \left\{\begin{array}{l}
\tilde{\nabla}_{S^{\prime}}=\Xi_{u^{\prime}} \frac{1}{i} \partial_{u^{\prime}}-\left[x^{\prime}, \frac{1}{i} \partial_{x^{\prime}}\right]-\left[v^{\prime}, \frac{1}{i} \partial_{v^{\prime}}\right] \\
\tilde{\nabla}_{P^{\prime}}=\frac{1}{i} \partial_{x^{\prime}}-\frac{1}{2} x^{\prime} \frac{1}{i} \partial_{v^{\prime}} \\
\tilde{\nabla}_{\Sigma^{\prime}}=\frac{1}{i} \partial_{v^{\prime}}
\end{array}\right.\right.
\end{aligned}
$$

In order to distinguish $\stackrel{\circ}{P}_{P}$ and $\tilde{\nabla}_{P}, u$ and $v$ are necessary.

Now let us write down concrete expression of the affine algebra generators, (3.3) and (3.4), in terms of (3.11), (3.12), (3.13) and (3.14) as the followings: 
- Covariant derivatives:

Flat left $\stackrel{\circ}{M}_{M}=\left(S_{m n}, P_{m}, \Sigma^{m n}\right)$

$$
\begin{aligned}
& \left\{\begin{array}{l}
S_{m n}=\Xi_{u} \frac{1}{i} \partial_{u} \\
P_{m}=\left(\beta^{-1 / 2}\left(\frac{1}{i} \partial_{x}-\frac{1}{2} \frac{1}{i} x \partial_{v}\right)+\frac{\alpha}{2} \beta^{1 / 2} \partial_{\sigma} x\right) e^{u} \\
\Sigma^{m n}=e^{-u}\left(\beta^{-1} \frac{1}{i} \partial_{v} e^{u}+\alpha \beta \partial_{\sigma} e^{u}\right)
\end{array}\right. \\
& \text { Flat right } \stackrel{\circ}{\nabla}_{M^{\prime}}=\left(S_{m^{\prime} n^{\prime}}, P_{m^{\prime}}, \Sigma^{m^{\prime} n^{\prime}}\right) \\
& \left\{\begin{array}{l}
S_{m^{\prime} n^{\prime}}=\Xi_{u^{\prime}} \frac{1}{i} \partial_{u^{\prime}} \\
P_{m^{\prime}}=\left(\beta^{-1 / 2}\left(\frac{1}{i} \partial_{x^{\prime}}+\frac{1}{2} \frac{1}{i} x^{\prime} \partial_{v^{\prime}}\right)-\frac{\alpha}{2} \beta^{1 / 2} \partial_{\sigma} x^{\prime}\right) e^{-u^{\prime}} \\
\Sigma^{m^{\prime} n^{\prime}}=e^{u^{\prime}}\left(\beta^{-1} \frac{1}{i} \partial_{v^{\prime}} e^{-u^{\prime}}+\alpha \beta \partial_{\sigma} e^{-u^{\prime}}\right)
\end{array}\right.
\end{aligned}
$$

- Symmetry generators:

Flat left $\tilde{\nabla}_{M}=\left(\tilde{S}_{m n} \tilde{P}_{m}, \tilde{\Sigma}^{m n}\right)$

$$
\left\{\begin{array}{l}
\tilde{S}_{m n}=\Xi_{-u} \frac{1}{i} \partial_{u}-x_{[m}\left(\frac{1}{i} \partial_{x}\right)_{n]}-v_{[m \mid}{ }^{l}\left(\frac{1}{i} \partial_{v}\right)_{n] l}-\alpha \partial_{\sigma} v \\
\tilde{P}_{m}=\beta^{-1 / 2}\left(\frac{1}{i} \partial_{x}+\frac{1}{2} \frac{1}{i} x \partial_{v}\right)-\frac{\alpha}{2} \beta^{1 / 2} \partial_{\sigma} x \\
\tilde{\Sigma}^{m n}=\beta^{-1} \frac{1}{i} \partial_{v}
\end{array}\right.
$$

Flat right $\tilde{\triangleright}_{M^{\prime}}=\left(\tilde{S}_{m^{\prime} n^{\prime}}, \tilde{P}_{m^{\prime}}, \tilde{\Sigma}^{m^{\prime} n^{\prime}}\right)$

$$
\left\{\begin{array}{l}
\tilde{S}_{m^{\prime} n^{\prime}}=\Xi_{u^{\prime}} \frac{1}{i} \partial_{u^{\prime}}+x_{\left[m^{\prime}\right.}\left(\frac{1}{i} \partial_{x^{\prime}}\right)_{\left.n^{\prime}\right]}+v_{\left[m^{\prime} \mid\right.} l^{\prime}\left(\frac{1}{i} \partial_{v^{\prime}}\right)_{\left.n^{\prime}\right] l^{\prime}}+\alpha \partial_{\sigma} v^{\prime} \\
\tilde{P}_{m^{\prime}}=\beta^{-1 / 2}\left(\frac{1}{i} \partial_{x^{\prime}}-\frac{1}{2} \frac{1}{i} x^{\prime} \partial_{v^{\prime}}\right)+\frac{\alpha}{2} \beta^{1 / 2} \partial_{\sigma} x^{\prime} \\
\tilde{\Sigma}^{m^{\prime} n^{\prime}}=\beta^{-1} \frac{1}{i} \partial_{v^{\prime}}
\end{array}\right.
$$

\subsection{Dimensional reduction constraints}

In this section the procedure of dimensional reduction of the doubled space into the usual space is presented. The doubled space is defined by the covariant derivatives $\triangleright_{\underline{M}}$ given in (3.3). Fields are functions of the doubled coordinates $Z \underline{M}$. This enlarged space contains auxiliary dimensions which are reduced if we impose the following constraints.

1. Section condition (strong constraint):

In the curved space covariant derivative operators are multiplied with the vielbein superfield $E_{\underline{A}} \underline{\underline{M}}[26-28,36]$. It can be orthonormal in the doubled space

$$
\triangleright_{\underline{A}}=E_{\underline{A}} \underline{M}^{\underline{M}} \stackrel{\circ}{M}_{\underline{M}}, \quad\left(E^{T}\right) \underline{M}_{\underline{A}} \eta^{\underline{A B}} E_{\underline{B}}{ }^{\underline{N}}=\eta \frac{M N}{} .
$$

The Virasoro operators (3.8) in curved space are given as

$$
\begin{aligned}
& \mathcal{H}_{\tau}=\frac{1}{2} \triangleright_{\underline{A}} \hat{\eta} \frac{A B}{{ }^{A}} \triangleright_{\underline{B}} \\
& \mathcal{H}_{\sigma}=\frac{1}{2} \triangleright_{\underline{A}} \eta \underline{\underline{A B}} \triangleright_{\underline{B}}=\frac{1}{2} \stackrel{\triangleright}{\triangleright}_{\underline{M}} \eta \underline{M N} \stackrel{\triangleright}{\triangleright}_{\underline{N}},
\end{aligned}
$$


and they are set to be zero as Virasoro constraints. $\mathcal{H}_{\sigma}=0$ is background field independent constraint in the doubled space. Section condition is the $\sigma$-diffeomorphism constraint $\mathcal{H}_{\sigma}=0$ in the second quantized level on all matrix elements as

$$
\begin{array}{r}
\mathcal{H}_{\sigma}=0 \Rightarrow \mathcal{H}_{\sigma}|\Phi\rangle=0 \text { and }\left\langle\Psi\left|\mathcal{H}_{\sigma}\right| \Phi\right\rangle=0 \\
\text { or } \\
\partial_{\underline{M}} \eta^{\underline{M N}} \partial_{\underline{N}} \Phi\left(Z^{\underline{M}}\right)=0 \text { and } \partial_{\underline{M}} \Psi\left(Z^{\underline{M}}\right) \eta^{\underline{M N}} \partial_{\underline{N}} \Phi\left(Z^{\underline{M}}\right)=0 .
\end{array}
$$

This constraint gives rise to the stringy contribution in the "new Lie derivative" [9-11, 26, 38].

\section{Isotropy constraints:}

For a coset group $\mathrm{G} / \mathrm{H}$, with $\mathrm{G}=$ Poincaré and $\mathrm{H}=$ Lorentz, Lorentz coordinates are suppressed by the isometry constraints $[26,30,31,36]$

$$
S_{m n}=S_{m^{\prime} n^{\prime}}=0 .
$$

These isotropy constraints are just particle covariant derivatives in (3.3), $\stackrel{\circ}{\nabla}_{S}=\stackrel{\circ}{\nabla}_{S^{\prime}}=$ 0 , for a constant solution $b_{I J}$ in (2.25). It allows gauging away the local Lorentz parameters.

3. Dimensional reduction constraints:

For nondegenerate pairs of generators $\left(S_{m n}, \Sigma^{m n}\right)$ and $\left(S_{m^{\prime} n^{\prime}}, \Sigma^{m^{\prime} n^{\prime}}\right), \Sigma^{m n}$ and $\Sigma^{m^{\prime} n^{\prime}}$ are auxiliary dimensions so these dimensions should be reduced. But $\Sigma^{m n}=$ $\Sigma^{m^{\prime} n^{\prime}}=0$ cannot be imposed as first class constraints, since they do not commute with the isotropy constraints (3.22). Instead symmetry generators can be imposed as first class constraints $[4,26,28,36]$

$$
\tilde{\Sigma}^{m n}=\tilde{\Sigma}^{m^{\prime} n^{\prime}}=0 .
$$

These constraints become covariant derivatives (3.4), and $\tilde{\nabla}_{\Sigma}=0=\stackrel{\circ}{\Sigma}_{\Sigma}=0$ from (3.13) and (3.14) for a constant solution $b_{I J}$ in (2.25). It allows gauging away $\Sigma$ and $\Sigma^{\prime}$ parameters.

4. Left/right mixing dimensional reduction constraint:

We also impose further dimensional reduction constraint which mixes the left and right sectors to reduce the doubled space into the usual space. Covariant derivatives $P_{m}$ and $P_{m^{\prime}}$ are dynamical degrees of freedom with the Virasoro constraints, while one combination of symmetry generators can be used as a first class constraint [36]

$$
\tilde{P}_{m}+\gamma \tilde{P}_{m^{\prime}}=0 \text {. }
$$

A commutation relation between constraints in (3.24) requires the dimensional reduction constraints $\tilde{\Sigma}^{m n}=\tilde{\Sigma}^{m^{\prime} n^{\prime}}=0$ in (3.23)

$$
\begin{aligned}
& {\left[\left(\tilde{P}_{m}+\gamma \tilde{P}_{m^{\prime}}\right)(1),\left(\tilde{P}_{n}+\gamma \tilde{P}_{n^{\prime}}\right)(2)\right]} \\
& \quad=i\left(\tilde{\Sigma}_{m n}+\gamma^{2} \tilde{\Sigma}_{m^{\prime} n^{\prime}}\right) \delta(2-1)+i\left(1-\gamma^{2}\right) \eta_{m n} \partial_{\sigma} \delta(2-1)
\end{aligned}
$$


where the Schwinger term cancels out only for $\gamma^{2}=1$. If the constraint is chosen as $\tilde{P}_{m}-\tilde{P}_{m^{\prime}}=0$ with $\gamma=-1$, then the sum of the left and right momenta corresponds to the total momentum for an usual space $\tilde{P}_{m}+\tilde{P}_{m^{\prime}}$.

Summarizing the above, dimensional reduction constraints for a bosonic string in the double coordinates space are isotropy constraints (3.22), dimensional reduction constraints (3.23) and the left/right mixing dimensional reduction constraint (3.24) in addition to the section constraint (3.21).

The two-dimensional diffeomorphisms are modified with the dimensional reduction constraints as

$$
\begin{aligned}
& \partial_{+} \Phi=i\left[\int d \sigma\left(\mathcal{A}+\mu S+\tilde{\mu} \tilde{\Sigma}+\tilde{\mu}^{P}\left(\tilde{P}+\gamma \tilde{P}^{\prime}\right)\right), \Phi\right] \\
& \partial_{-} \Phi=i\left[\int d \sigma\left(\mathcal{A}^{\prime}+\mu^{\prime} S^{\prime}+\tilde{\mu}^{\prime} \tilde{\Sigma}^{\prime}+\tilde{\mu}^{P}\left(\tilde{P}+\gamma \tilde{P}^{\prime}\right)\right), \Phi\right] .
\end{aligned}
$$

The covariant derivatives are still manifestly chiral up to the trivial Lorentz rotation

$$
\partial_{-} \stackrel{\triangleright}{\triangleright}_{M}=0=\partial_{+} \stackrel{\triangleright}{\triangleright}_{M^{\prime}}
$$

but not the symmetry generators in general.

Now we go back to the T-duality symmetry. Let us examine the global $\mathrm{O}(\mathrm{n}, \mathrm{n})$ transformation on constraints. The section condition $\mathcal{H}_{\sigma}=0$ is inert under the global $\mathrm{O}(\mathrm{n}, \mathrm{n})$. The dimensional reduction constraints $\tilde{\Sigma}^{m n}=\tilde{\Sigma}^{m^{\prime} n^{\prime}}=\tilde{P}_{m}+\gamma \tilde{P}_{m^{\prime}}=0$ are also inert under the global $\mathrm{O}(\mathrm{n}, \mathrm{n})$ transformation as shown in (2.46). The isotropy constraints are transformed under $\mathrm{O}(\mathrm{n}, \mathrm{n}) \ni \mathcal{O}_{\underline{M}}{ }^{\underline{N}}$; so we must impose the consistency condition as

$$
S_{m n}=S_{m^{\prime} n^{\prime}}=0, \quad \delta \check{\triangleright}_{\underline{S}}=\mathcal{O}_{\underline{S}} \underline{M}^{\triangleright^{\circ}} \underline{M}_{M}=0 .
$$

The dimensional reduction constraints $\tilde{\Sigma}^{m n}=\tilde{\Sigma}^{m^{\prime} n^{\prime}}=0$ coincide with $\Sigma^{m n}=\Sigma^{m^{\prime} n^{\prime}}=0$ in the unitary gauge (3.3) and (3.4). Then analogously to the above

$$
\Sigma^{m n}=\Sigma^{m^{\prime} n^{\prime}}=0, \quad \delta \stackrel{\circ}{\Sigma}_{\underline{\Sigma}}=\mathcal{O}_{\underline{\Sigma}} \underline{M}^{\grave{\triangleright}^{M}}=0 .
$$

From the orthonormal property an $\mathrm{O}(\mathrm{n}, \mathrm{n})$ matrix must satisfies

$$
\mathcal{O}_{\underline{S} \underline{M}}=\mathcal{O}_{\underline{\Sigma} \underline{M}}=\mathcal{O}_{\underline{M}} \underline{S}=\mathcal{O}_{\underline{M}} \underline{\Sigma}=0 .
$$

As a result the survived symmetry is $\mathrm{O}(\mathrm{d}, \mathrm{d}), \mathcal{O}_{\underline{P P}}$, which is the T-duality symmetry in the usual space.

\subsection{Bosonic string action}

The Hamiltonian for a bosonic string in the doubled space is given by

$$
\begin{aligned}
\mathcal{H}= & \lambda_{\tau} \mathcal{H}_{\tau}+\lambda_{\sigma} \mathcal{H}_{\sigma}+\mu^{m n} S_{m n}+\mu^{m^{\prime} n^{\prime}} S_{m^{\prime} n^{\prime}}+\tilde{\mu}_{m n} \tilde{\Sigma}^{m n}+\tilde{\mu}_{m^{\prime} n^{\prime}} \tilde{\Sigma}^{m^{\prime} n^{\prime}} \\
& +\tilde{\mu}^{m}\left(\tilde{P}_{m}-\tilde{P}_{m^{\prime}}\right) \\
= & \frac{1}{2} \stackrel{\triangleright}{ }_{\underline{M}}\left(\lambda_{\tau} \hat{\eta} \underline{\underline{M N}}+\lambda_{\sigma} \eta \underline{M N}\right) \stackrel{\triangleright}{\triangleright}_{\underline{N}}+\mu^{\underline{M}} \stackrel{\triangleright}{\Phi}_{\underline{M}}+\tilde{\mu} \underline{\underline{M}} \tilde{\triangleright}_{\underline{M}}
\end{aligned}
$$


with

$$
\begin{aligned}
& \mu^{\underline{M}}=\left(\mu^{S}, \mu^{S^{\prime}}, \text { others }=0\right) . \\
& \tilde{\mu}^{\underline{M}}=\left(\tilde{\mu}^{\Sigma}, \tilde{\mu}^{\Sigma^{\prime}}, \tilde{\mu}^{P}, \tilde{\mu}^{P^{\prime}}=-\tilde{\mu}^{P}, \text { others }=0\right) .
\end{aligned}
$$

An action for the bosonic string is given by

$$
I=\int d \tau d \sigma \mathcal{L}, \quad \mathcal{L}=\partial_{\tau} Z^{\underline{M}} \frac{1}{i} \partial_{\underline{M}}-\mathcal{H}=J_{0} \underline{\underline{M}} \stackrel{\circ}{\nabla}_{\underline{M}}-\mathcal{H} .
$$

In this section we obtain an gauge invariant action without specifying a solution for $B$ field. From (2.21) and (2.25) we use

$$
\begin{aligned}
& \stackrel{\triangleright}{\triangleright}_{M}=\stackrel{\circ}{\nabla}_{M}+J_{1}^{K} b_{K M}=\stackrel{\circ}{\nabla}_{M}+\frac{1}{2} J_{1}^{K}\left(\eta_{K M}+B_{K M}\right) \\
& \tilde{\triangleright}_{M}=\tilde{\nabla}_{M}+\tilde{J}_{1}^{K} \tilde{b}_{K M}=M_{M}^{N}\left(\stackrel{\triangleright}{\triangleright}_{N}-J_{1}^{K} \eta_{K N}\right)
\end{aligned}
$$

and the similar relations for the right sector. In general $M_{I}^{J}=\left(L^{-1} R\right)_{I}^{J}$ is triangular matrix from the relation of canonical dimensions. The orthonormality of $M_{I}^{J}$ leads to the orthonormality of $M_{P}{ }^{P}$

$$
M_{I}^{J}=\begin{gathered}
S \\
P \\
\Sigma
\end{gathered}\left(\begin{array}{ccc}
S & P & \Sigma \\
* & * & * \\
0 & * & * \\
0 & 0 & *
\end{array}\right) \Rightarrow M_{P_{l}}{ }^{P_{m}} M_{P_{k}}^{P_{n}} \eta_{P_{m} P_{n}}=\eta_{P_{l} P_{k}}
$$

where $*$ 's denote nonzero elements. The matrices $M_{P} P$ and $M_{P^{\prime}} P^{\prime}$ are Lorentz rotation matrices, and they are functions of only Lorentz parameters with the canonical dimension 0 . The triangular property of $M_{I}^{J}$ leads to

$$
\begin{aligned}
\tilde{P} & =M_{P}{ }^{P}\left(P-J_{1}^{P}\right)+M_{P}{ }^{\Sigma}\left(M_{\Sigma}{ }^{\Sigma}\right)^{-1} \tilde{\Sigma} \\
\tilde{P}^{\prime} & =M_{P^{\prime}} P^{\prime}\left(P^{\prime}+J_{1}^{P^{\prime}}\right)+M_{P^{\prime}} \Sigma^{\prime}\left(M_{\Sigma^{\prime}}{ }^{\Sigma^{\prime}}\right)^{-1} \tilde{\Sigma}^{\prime} \\
\stackrel{\circ}{\nabla}_{\Sigma} & =\frac{1}{2} J_{1}{ }^{S}-\frac{1}{2} J_{1}{ }^{K} B_{K \Sigma}+\left(M_{\Sigma}{ }^{\Sigma}\right)^{-1} \tilde{\Sigma} .
\end{aligned}
$$

Using (3.34) and (3.36) the Lagrangian becomes sum of the kinetic part $\mathcal{L}_{0}$, the Wess-Zumino terms $\mathcal{L}_{W Z}$, the boundary of the Wess-Zumino terms $\mathcal{L}_{W Z ; 0}$ and constraint part $\mathcal{L}_{\text {const }}$ :

$$
\begin{aligned}
& \mathcal{L}=\mathcal{L}_{0}+\mathcal{L}_{W Z}+\mathcal{L}_{W Z ; 0}+\mathcal{L}_{\text {const }} \\
& \left\{\begin{aligned}
\mathcal{L}_{0}= & J_{0}{ }^{P} P-\frac{\lambda}{2} P^{2}-\frac{1}{2} J_{0}{ }^{P} J_{1}{ }^{P}-\tilde{\mu}^{P} M_{P} P\left(P-J_{1}{ }^{P}\right) \\
& +J_{0} P^{\prime} P^{\prime}-\frac{\lambda^{\prime}}{2} P^{\prime 2}+\frac{1}{2} J_{0} P^{\prime} J_{1} P^{\prime}+\tilde{\mu}^{P} M_{P^{\prime}} P^{\prime}\left(P^{\prime}+J_{1} P^{\prime}\right) \\
\mathcal{L}_{W Z}= & -\frac{1}{2}\left(J_{0}{ }^{N} J_{1}{ }^{M} B_{M N}-J_{0}{ }^{\prime} J_{1}{ }^{M^{\prime}} B_{M^{\prime} N^{\prime}}\right) \\
\mathcal{L}_{W Z ; 0}= & \frac{1}{2}\left(J_{[0}{ }^{\Sigma} J_{1]}{ }^{S}-J_{[0} \Sigma^{\prime} J_{1]}{ }^{S^{\prime}}\right) \\
\mathcal{L}_{\text {const }}= & -\hat{\mu}^{S} S-\hat{\mu}^{S^{\prime}} S^{\prime}-\hat{\mu}^{\Sigma} \tilde{\Sigma}-\hat{\mu}^{\Sigma^{\prime} \tilde{\Sigma}^{\prime}}
\end{aligned}\right.
\end{aligned}
$$


by redefining Lagrange multipliers as

$$
\left\{\begin{aligned}
\lambda & =\lambda_{\tau}+\lambda_{\sigma}, & \lambda^{\prime} & =\lambda_{\tau}-\lambda_{\sigma} \\
\hat{\mu}^{S} & =\mu^{S}-J_{0}^{S}+\frac{\lambda}{2} \Sigma, & \hat{\mu}^{S^{\prime}} & =\mu^{S^{\prime}}-J_{0}^{S^{\prime}}+\frac{\lambda^{\prime}}{2} \Sigma^{\prime} \\
\hat{\mu}^{\Sigma} & \left.=\tilde{\mu}^{\Sigma}-\left(J_{0}^{\Sigma}-\tilde{\mu}^{P} M_{P}{ }^{\Sigma}\right)\left(M_{\Sigma}\right)^{\Sigma}\right)^{-1}, & \hat{\mu}^{\Sigma^{\prime}} & =\tilde{\mu}^{\Sigma^{\prime}}-\left(J_{0}^{\Sigma^{\prime}}+\tilde{\mu}^{P} M_{P^{\prime}} \Sigma^{\prime}\right)\left(M_{\Sigma^{\prime}}{ }^{\Sigma^{\prime}}\right)^{-1}
\end{aligned}\right.
$$

Variation of the action with respect to $\tilde{\mu}^{P}$ gives the left/right mixed dimensional reduction constraint

$$
\tilde{P}^{\prime}-\tilde{P}=\left(P^{\prime}+J_{1}{ }^{\prime}\right)-\underline{M}_{P^{\prime}}{ }^{P}\left(P-J_{1}{ }^{P}\right)=0, \quad \underline{M}_{P^{\prime}}^{P}=\left(M_{P^{\prime}}{ }^{\prime}\right)^{-1} M_{P}{ }^{P} .
$$

$\underline{M}_{P^{\prime}}{ }^{P}$ is a Lorentz rotation matrix which relate the left and the right spaces. A similar matrix is introduced in [34]. After integrating out both $P$ and $P^{\prime}$ the kinetic part becomes

$$
\begin{aligned}
\mathcal{L}_{0} & =\frac{\beta}{2\left(\lambda+\lambda^{\prime}\right)}\left[\left(J_{0}^{\mathcal{P}+}\right)^{2}-\alpha^{2} \lambda \lambda^{\prime}\left(J_{1}^{\mathcal{P}+}\right)^{2}+\alpha\left(-\lambda+\lambda^{\prime}\right) J_{0}^{\mathcal{P}+} J_{1}^{\mathcal{P}+}\right]+\frac{\alpha \beta}{2} J_{[0}^{P} J_{1]}^{P^{\prime}} \underline{M}_{P^{\prime}}{ }^{P} \\
J_{i}^{\mathcal{P} \pm} & =\frac{1}{\sqrt{2}}\left(J_{i}^{P} \pm J_{i}^{P^{\prime}} \underline{M}_{P^{\prime}}^{P}\right)
\end{aligned}
$$

where $\alpha$ and $\beta$ are parameters for normalization in (3.6) and (3.7). Two Lagrange multipliers correspond to the worldsheet metric as

$$
\alpha \lambda=-\frac{h^{01}}{h^{00}}+\frac{1}{\sqrt{-h} h^{00}}, \quad \alpha \lambda^{\prime}=\frac{h^{01}}{h^{00}}+\frac{1}{\sqrt{-h} h^{00}} .
$$

As a result the gauge invariant action for a bosonic string based on the double nondegenerate Poincaré algebra is given by

$$
\begin{array}{r}
I=\int d \tau d \sigma \mathcal{L}, \quad \mathcal{L}=\mathcal{L}_{0}+\mathcal{L}_{W Z}+\mathcal{L}_{W Z ; 0} \\
\left\{\begin{array}{l}
\mathcal{L}_{0} \quad=\frac{\alpha \beta}{2}\left[\sqrt{-h} h^{i j} J_{i}^{\mathcal{P}+} J_{j}^{\mathcal{P}+}-\epsilon^{i j} J_{i}^{\mathcal{P}+} J_{j}^{\mathcal{P}-}\right] \\
\mathcal{L}_{W Z}=-\frac{\alpha \beta}{2}\left(J_{0}{ }^{N} J_{1}{ }^{M} B_{M N}-J_{0}{ }^{\prime \prime} J_{1}{ }^{M^{\prime}} B_{M^{\prime} N^{\prime}}\right) \\
\mathcal{L}_{W Z ; 0}=\frac{\alpha \beta}{2}\left(J_{[0}{ }^{\Sigma} J_{1]}{ }^{S}-J_{[0}{ }^{\prime} J_{1]}{ }^{S^{\prime}}\right)
\end{array}\right.
\end{array}
$$

If we impose the simple solution for $B$ field in (2.25), the Wess-Zumino term $\mathcal{L}_{W Z}$ is cancelled out by the boundary term of the Wess-Zumino term $\mathcal{L}_{W Z ; 0}$. For a general solution of $B$ field $\mathcal{L}_{W Z}+\mathcal{L}_{W Z ; 0}$ becomes total derivative term.

Taking variation of the action (3.42) with respect to $Z^{\underline{M}}$ the following first class constraints are derived; the Virasoro constraints in (3.8), isotropy constraints (3.22), dimensional reduction constraints (3.23) and the left/right mixing dimensional reduction constraint (3.24)

$$
\mathcal{H}_{\tau}=\mathcal{H}_{\sigma}=S_{m n}=S_{m^{\prime} n^{\prime}}=\tilde{\Sigma}^{m n}=\tilde{\Sigma}^{m^{\prime} n^{\prime}}=\tilde{P}_{m}-\tilde{P}_{m^{\prime}}=0 .
$$

The gauge invariance generated by the above first class constraints is preserved. 


\subsection{Gauge fixing}

Corresponding to the first class constraints (3.43) we can choose the following gauge fixing conditions. For the isotropy constraints (3.22) and the dimensional reduction constraints $(3.23)$

$$
S_{m n}=S_{m^{\prime} n^{\prime}}=0, \quad \tilde{\Sigma}^{m n}=\tilde{\Sigma}^{m^{\prime} n^{\prime}}=0,
$$

the simplest gauge is an unitary gauge

$$
u^{m n}=u^{m^{\prime} n^{\prime}}=0, \quad v_{m n}=v_{m^{\prime} n^{\prime}}=\text { const. . }
$$

Let us introduce two kinds of coordinates as

$$
\begin{aligned}
X^{\mathrm{m}} & =\frac{1}{\sqrt{2}}\left(x^{m}+x^{m^{\prime}}\right), & Y_{\mathrm{m}} & =\frac{1}{\sqrt{2}}\left(x^{m}-x^{m^{\prime}}\right), \\
\frac{\partial}{\partial X^{\mathrm{m}}} & =\frac{1}{\sqrt{2}}\left(\partial_{x^{m}}+\partial_{x^{m^{\prime}}}\right), & \frac{\partial}{\partial Y_{\mathrm{m}}} & =\frac{1}{\sqrt{2}}\left(\partial_{x^{m}}-\partial_{x^{m^{\prime}}}\right)
\end{aligned}
$$

where $X^{\mathrm{m}}$ and $Y_{\mathrm{m}}$ correspond to the usual space coordinate and the dual coordinate respectively. The unitary gauge (3.44) allows $\tilde{J}^{S}=\tilde{J}^{S^{\prime}}=0, \quad \tilde{\nabla}_{P}=\stackrel{\circ}{\nabla}_{P}, \quad \tilde{\nabla}_{P^{\prime}}=\stackrel{\circ}{\nabla}_{P^{\prime}}, \quad \tilde{J}^{P}=J^{P}$ and $\tilde{J}^{P^{\prime}}=J^{P^{\prime}}$. The momentum operators are rewritten from (3.17) and (3.18) in the unitary gauge in terms of $X^{\mathrm{m}}$ and $Y_{\mathrm{m}}$ as

$$
\left\{\begin{array} { l } 
{ P _ { m } + P _ { m ^ { \prime } } = \frac { \sqrt { 2 } } { i } \frac { \partial } { \partial X ^ { \mathrm { m } } } + \frac { 1 } { \sqrt { 2 } } \partial _ { \sigma } Y _ { \mathrm { m } } } \\
{ P _ { m } - P _ { m ^ { \prime } } = \frac { \sqrt { 2 } } { i } \frac { \partial } { \partial Y _ { \mathrm { m } } } + \frac { 1 } { \sqrt { 2 } } \partial _ { \sigma } X ^ { \mathrm { m } } }
\end{array} \quad \left\{\begin{array}{l}
\tilde{P}_{m}+\tilde{P}_{m^{\prime}}=\frac{\sqrt{2}}{i} \frac{\partial}{\partial X^{\mathrm{m}}}-\frac{1}{\sqrt{2}} \partial_{\sigma} Y_{\mathrm{m}} \\
\tilde{P}_{m}-\tilde{P}_{m^{\prime}}=\frac{\sqrt{2}}{i} \frac{\partial}{\partial Y_{\mathrm{m}}}-\frac{1}{\sqrt{2}} \partial_{\sigma} X^{\mathrm{m}}
\end{array} .\right.\right.
$$

They become the usual "dual coordinate relation" $\tilde{P}_{m} \pm \tilde{P}_{m^{\prime}} \Leftrightarrow \partial_{i} Y_{\mathrm{m}}-\epsilon_{i j} \partial^{j} X^{\mathrm{m}}$ in a flat space. Contrast to the conventional relation $\partial_{i} Y_{\mathrm{m}}-\epsilon_{i j} \partial^{j} X^{\mathrm{m}}=0$ [5-7], we set only one of them to be zero, $\tilde{P}-\tilde{P}^{\prime}=0$. This is the left/right mixed dimensional reduction constraint which allows the following gauge fixing condition on $Y_{\mathrm{m}}$.

$$
\tilde{P}_{m}-\tilde{P}_{m^{\prime}}=\frac{\sqrt{2}}{i} \frac{\partial}{\partial Y_{\mathrm{m}}}-\frac{1}{\sqrt{2}} \partial_{\sigma} X^{\mathrm{m}}=0 \Rightarrow \partial_{\sigma} Y_{\mathrm{m}}=0 .
$$

Then the momentum operators become

$$
\left\{\begin{array} { l } 
{ P _ { m } = \frac { 1 } { \sqrt { 2 } } ( \frac { 1 } { i } \frac { \partial } { \partial X ^ { \mathrm { m } } } + \partial _ { \sigma } X ^ { \mathrm { m } } ) } \\
{ P _ { m ^ { \prime } } = \frac { 1 } { \sqrt { 2 } } ( \frac { 1 } { i } \frac { \partial } { \partial X ^ { \mathrm { m } } } - \partial _ { \sigma } X ^ { \mathrm { m } } ) }
\end{array} \quad \left\{\begin{array}{l}
\tilde{P}_{m}+\tilde{P}_{m^{\prime}}=\frac{\sqrt{2}}{i} \frac{\partial}{\partial X^{\mathrm{m}}} \\
\tilde{P}_{m}-\tilde{P}_{m^{\prime}}=0
\end{array}\right.\right.
$$

where $P_{m}$ and $P_{m^{\prime}}$ are the left and right moving modes in the usual space and the $\tilde{P}_{m}+\tilde{P}_{m^{\prime}}$ is the total momentum of the space.

The section condition (3.21) becomes simpler in the unitary gauge

$$
\mathcal{H}_{\sigma} \approx\left(\frac{1}{i} \frac{\partial}{\partial X}+\frac{1}{2} \partial_{\sigma} Y\right)_{\mathrm{m}}\left(\frac{1}{i} \frac{\partial}{\partial Y}+\frac{1}{2} \partial_{\sigma} X\right)^{\mathrm{m}}=\frac{1}{i} \frac{\partial}{\partial X}{ }_{\mathrm{m}} \partial_{\sigma} X^{\mathrm{m}}=0
$$


where $\approx$ uses the local Lorentz constraints (isotropy constraints). A solution in the second quantized level of (3.46) is given by $\Psi(X, Y)=e^{\frac{i}{2} \int Y \cdot \partial_{\sigma} X} \Phi(X)$. The section condition reduces to the usual $\sigma$ diffeomorphism constraint (3.48) in the simple gauge (3.46) as $\frac{\partial}{\partial X}{ }^{\mathrm{m}} \partial_{\sigma} X^{\mathrm{m}} \Phi(X)=0$.

There is another simple gauge $u=u^{\prime}$ in such a way that $M_{P^{\prime}}{ }^{P}=1$, which gives $J_{i}^{\mathcal{P} \pm}=J_{i}^{P} \pm J_{i}^{P^{\prime}}$. In the unitary gauge $u=u^{\prime}=0$ they become very simple with the usual space coordinates $(3.45) ; J_{i}^{\mathcal{P}+}=\partial_{i} X$ and $J_{i}^{\mathcal{P}-}=\partial_{i} Y$. The Lagrangian for a bosonic string in an unitary gauge $u=u^{\prime}=0$ is rewritten as

$$
\mathcal{L}_{0}=d X \wedge * d X-d X \wedge d Y
$$

The second term in (3.49) including the dual coordinate is a total derivative in a flat space. This term gives the first class constraint $\frac{\partial}{i \partial Y^{\mathrm{m}}}-\frac{1}{2} \partial_{\sigma} X^{\mathrm{m}}=0$ in (3.46). Further simple gauge for the section condition (3.21), $Y_{\mathrm{m}}=0$, the action reduces to the usual bosonic string action.

\section{Superstring action in doubled space}

In this section we construct an gauge invariant action for the type II superstring in the doubled space. At first we present the chiral affine super-Poincaré algebras. The dimensional reduction constraints are extended for a supersymmetric case. Then we write down an gauge invariant action without using a specific solution of $B$-field.

\subsection{Doubled chiral super-Poincaré generators}

For a superstring we use a nondegenerate super-Poincaré algebra generated by $G_{I}=$ $\left(s_{m n}, d_{\mu}, p_{m}, \omega^{\mu}, \sigma^{m n}\right)$ with canonical dimensions $\left(0, \frac{1}{2}, 1, \frac{3}{2}, 2\right)$ respectively. The algebra is given as

$$
\begin{aligned}
& \operatorname{dim} 0: \quad\left[s_{m n}, s_{l k}\right]=-i \eta_{[k \mid[m} s_{n] \mid l]} \\
& \operatorname{dim} \frac{1}{2}: \quad\left[s_{m n}, d_{\mu}\right]=-\frac{i}{2}\left(d \gamma_{m n}\right)_{\mu} \\
& \operatorname{dim} 1: \quad\left[s_{m n}, p_{l}\right]=-i p_{[m} \eta_{n] l}, \quad\left\{d_{\mu}, d_{\nu}\right\}=2 p_{m} \gamma^{m}{ }_{\mu \nu} \\
& \operatorname{dim} \frac{3}{2}: \quad\left[s_{m n}, \omega^{\mu}\right]=\frac{i}{2}\left(\gamma_{m n} \omega\right)^{\mu}, \quad\left[d_{\mu}, p_{m}\right]=2\left(\gamma_{n} \omega\right)_{\mu} \\
& \operatorname{dim} 2: \quad\left[s_{m n}, \sigma^{l k}\right]=-i \delta_{[m}^{[k} \sigma_{n]}^{l]}, \quad\left\{d_{\mu}, \omega^{\nu}\right\}=-\frac{i}{4} \sigma^{m n}\left(\gamma_{m n}\right)^{\nu}{ }_{\mu},\left[p_{m}, p_{n}\right]=i \sigma_{m n} .
\end{aligned}
$$

The nondegenerate group metric is

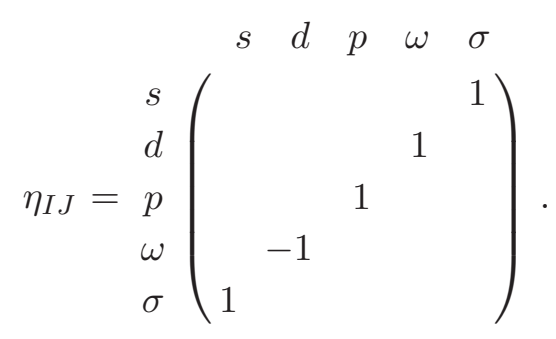


The nondegenerate super-Poincaré algebra (4.1) is extended to double affine Lie algebras. The covariant derivatives and the symmetry generators (2.26) are given as follows:

- Covariant derivatives:

Flat left

$$
\begin{aligned}
& {\stackrel{\triangleright}{\triangleright_{M}}}=\left(S_{m n}, D_{\mu}, P_{m}, \Omega^{\mu}, \Sigma^{m n}\right) \\
& \left\{\begin{aligned}
S_{m n} & =\stackrel{\circ}{\nabla}_{S} \\
D_{\mu} & =\stackrel{\circ}{\nabla}_{D}-\frac{1}{4} J_{1}^{\Omega} \\
P_{m} & =\stackrel{\circ}{\nabla}_{P}+\frac{1}{2} J_{1}^{P} \\
\Omega^{\mu} & =\stackrel{\circ}{\nabla}_{\Omega}+\frac{3}{4} J_{1}^{D} \\
\Sigma^{m n} & =\stackrel{\circ}{\nabla}_{\Sigma}+J_{1}^{S}
\end{aligned}\right.
\end{aligned}
$$

: Flat right

$$
{\stackrel{\circ}{M^{\prime}}}_{M^{\prime}}=\left(S_{m^{\prime} n^{\prime}}, D_{\mu^{\prime}}, P_{m^{\prime}}, \Omega^{\mu^{\prime}}, \Sigma^{m^{\prime} n^{\prime}}\right)
$$

$$
\left\{\begin{aligned}
& S_{m^{\prime} n^{\prime}}=\stackrel{\circ}{\nabla}_{S^{\prime}} \\
& D_{\mu^{\prime}}=\stackrel{\circ}{\nabla}_{D^{\prime}}+\frac{1}{4} J_{1}^{\Omega^{\prime}} \\
& P_{m^{\prime}}=\stackrel{\circ}{\nabla}_{P^{\prime}}-\frac{1}{2} J_{1}^{P^{\prime}} \\
& \Omega^{\mu^{\prime}}=\circ^{\circ} \\
& \Sigma_{\Omega^{\prime}}-\frac{3}{4} J_{1}^{D^{\prime}} \\
& \Sigma^{m^{\prime} n^{\prime}}=\stackrel{\circ}{\nabla}_{\Sigma^{\prime}}-J_{1}^{S^{\prime}}
\end{aligned}\right.
$$

- Symmetry generators:

$$
\begin{aligned}
& \text { Flat left } \tilde{\triangleright}_{M}=\left(\tilde{S}_{m n}, \tilde{D}_{\mu}, \tilde{P}_{m}, \tilde{\Omega}^{\mu}, \tilde{\Sigma}^{m n}\right) \\
& \left\{\begin{aligned}
\tilde{S}_{m n} & =\tilde{\nabla}_{S}-\left(\tilde{J}_{1}^{\Sigma}+\sum_{N=S, D, P, \Omega} c_{S}^{N} \tilde{J}_{1}^{N}\right) \\
\tilde{D}_{\mu} & =\tilde{\nabla}_{D}+\frac{3}{4}\left(\tilde{J}_{1}^{\Omega}+\sum_{N=S, D, P} c_{D}^{N} \tilde{J}_{1}^{N}\right) \\
\tilde{P}_{m} & =\tilde{\nabla}_{P}-\frac{1}{2}\left(\tilde{J}_{1}^{P}+\sum_{N=S, D} c_{P}^{N} \tilde{J}_{1}^{N}\right) \\
\tilde{\Omega}^{\mu} & =\tilde{\nabla}_{\Omega}-\frac{1}{4}\left(\tilde{J}_{1}^{D}+c_{\Omega}^{S} \tilde{J}_{1}^{S}\right) \\
\tilde{\Sigma}^{m n} & =\tilde{\nabla}_{\Sigma}
\end{aligned}\right. \\
& \text { Flat right } \tilde{\nabla}_{M^{\prime}}=\left(\tilde{S}_{m^{\prime} n^{\prime}}, \tilde{D}_{\mu^{\prime}}, \tilde{P}_{m^{\prime}}, \tilde{\Omega}^{\mu^{\prime}}, \tilde{\Sigma}^{m^{\prime} n^{\prime}}\right) \\
& \left\{\begin{aligned}
\tilde{S}_{m^{\prime} n^{\prime}} & =\tilde{\nabla}_{S^{\prime}}+\left(\tilde{J}_{1}^{\Sigma^{\prime}}+\sum_{N^{\prime}=S^{\prime}, D^{\prime}, P^{\prime}, \Omega^{\prime}} c_{S^{\prime}}^{N^{\prime}} \tilde{J}_{1}^{N^{\prime}}\right) \\
\tilde{D}_{\mu^{\prime}} & =\tilde{\nabla}_{D^{\prime}}-\frac{3}{4}\left(\tilde{J}_{1}^{\Omega^{\prime}}+\sum_{N^{\prime}=S^{\prime}, D^{\prime}, P^{\prime}} c_{D^{\prime}}^{N^{\prime}} \tilde{J}_{1}^{N^{\prime}}\right. \\
\tilde{P}_{m^{\prime}} & =\tilde{\nabla}_{P^{\prime}}+\frac{1}{2}\left(\tilde{J}_{1}^{P^{\prime}}+\sum_{N^{\prime}=S^{\prime}, D^{\prime}} c_{P^{\prime}}^{N^{\prime}} \tilde{J}_{1}^{N^{\prime}}\right) \\
\tilde{\Omega}^{\mu^{\prime}} & =\tilde{\nabla}_{\Omega^{\prime}}+\frac{1}{4}\left(\tilde{J}_{1}^{D^{\prime}}+c_{\Omega^{\prime}}^{S^{\prime}} \tilde{J}_{1}^{S^{\prime}}\right) \\
\tilde{\Sigma}^{m^{\prime} n^{\prime}} & =\tilde{\nabla}_{\Sigma^{\prime}}
\end{aligned}\right.
\end{aligned}
$$

Coefficients $c_{M}^{N}$ in symmetry generators are determined from $\tilde{J}_{1}^{N} \tilde{b}_{N M}$ terms in $(2.23)$ and (2.24) and they are functions of super-coordinates with canonical dimensions $n_{M}+$ $n_{N}-2$. From the nondegeneracy of the group (4.3) and (4.4) are unique representation of the affine nondegenerate algebras (2.18) and (2.19) with the superspace metric (4.2) up to 
the rescaling of currents. Rescaling currents with parameters $\alpha$ and $\beta$, in such a way that they satisfy the algebra (3.6), is given as

$$
\left\{\begin{array}{rl}
S_{m n} & =\stackrel{\circ}{\nabla}_{S} \\
D_{\mu} & =\beta^{-1 / 4} \stackrel{\circ}{\nabla}_{D}-\frac{1}{4} \alpha \beta^{3 / 4} J_{1}^{\Omega} \\
P_{m} & =\beta^{-1 / 2} \stackrel{\circ}{\nabla}_{P}+\frac{1}{2} \alpha \beta^{1 / 2} J_{1}^{P} \\
\Omega^{\mu} & =\beta^{-3 / 4} \stackrel{\circ}{\nabla}_{\Omega}+\frac{3}{4} \alpha \beta^{1 / 4} J_{1}^{D} \\
\Sigma^{m n} & =\beta^{-1} \stackrel{\circ}{\nabla}_{\Sigma}+\alpha \beta J_{1}^{S}
\end{array} .\right.
$$

The same rescaling is done for other sectors.

\subsection{Superstring action}

In this section the supersymmetric extension of the section 3 is presented. The doubled space is defined by the supercovariant derivatives $\stackrel{\circ}{M}_{\underline{M}}$ which are given in (4.3) and parameterized by doubled super-coordinates $Z^{M}$. This enlarged space contains auxiliary dimensions which are reduced by a set of first class constraints as same as the bosonic case: 1. the section condition (strong constraints), 2. isotropy constraints and 4. Left/right mixing dimensional constraint are the same as before. Only the condition 3. dimensional reduction constraints for fermions are added.

3. Dimensional reduction constraints:

In order to describe correct physical degrees of freedom unphysical dimensions introduced by nondegeneracy of the group are eliminated. In order to preserve isotropy constraints and the $\kappa$-symmetry, symmetry generator currents are chosen to be constraints to reduce auxiliary dimensions as $[26,28,36]$

$$
\tilde{\triangleright}_{\underline{M}}=0 \text { for } n_{\underline{M}}>1 \Leftrightarrow \tilde{\Sigma}^{m n}=\tilde{\Sigma}^{m^{\prime} n^{\prime}}=\tilde{\Omega}^{\mu}=\tilde{\Omega}^{\mu^{\prime}}=0 .
$$

For superstrings the Virasoro constraints are extended to the $\kappa$-symmetric Virasoro constraints $\mathcal{A B C D}$ constraints $[3,28,30,31]$ as

$$
\begin{aligned}
\mathcal{A} & =\frac{1}{2} P_{m} P^{m}+\Omega^{\mu} D_{\mu}+\frac{1}{2} \Sigma^{m n} S_{m n}=0 \\
\mathcal{B}^{\mu} & =P_{m}\left(\gamma^{m} D\right)^{\mu}-i S_{m n}\left(\gamma^{m n} \Omega\right)^{\mu}=0 \\
\mathcal{C}_{\mu \nu} & =D_{\mu} D_{\nu}+\frac{1}{2 i} S_{m n} P_{l}\left(\gamma^{m n l}\right)_{\mu \nu}=0 \\
\mathcal{D}_{m} & =D \gamma_{m} \partial_{\sigma} D+\frac{4}{i} \Sigma_{m n} S^{n l} P_{l}=0 .
\end{aligned}
$$

The same set of constraints for the right sector. First class constraints for a type II superstring in a flat space are $\mathcal{A B C D}$ constraints (4.7), isotropy constraints (3.22), dimensional reduction constraints (3.23) and the left/right mixing dimensional reduction constraint (3.24)

$$
\mathcal{A}=\mathcal{B}=\mathcal{C}=\mathcal{D}=S_{m n}=\tilde{\Sigma}^{m n}=\tilde{\Omega}^{\mu}=\tilde{P}_{m}-\tilde{P}_{m^{\prime}}=0
$$

and the similar constraints for the right sector. 
The Hamiltonian for a type II superstring in T-duality covariant form is given by

$$
\begin{aligned}
\mathcal{H}= & \lambda \mathcal{A}+\lambda_{\mu} \mathcal{B}^{\mu}+\lambda^{\mu \nu} \mathcal{C}_{\mu \nu}+\lambda^{m} \mathcal{D}_{m}+\mu^{m n} S_{m n}+\tilde{\mu}_{\mu} \tilde{\Omega}^{\mu}+\tilde{\mu}_{m n} \tilde{\Sigma}^{m n} \\
& +\lambda^{\prime} \mathcal{A}+\lambda_{\mu^{\prime}} \mathcal{B}^{\mu^{\prime}}+\lambda^{\mu^{\prime} \nu^{\prime}} \mathcal{C}_{\mu^{\prime} \nu^{\prime}}+\lambda^{m^{\prime}} \mathcal{D}_{m^{\prime}}+\mu^{m^{\prime} n^{\prime}} S_{m^{\prime} n^{\prime}}+\tilde{\mu}_{\mu^{\prime}} \tilde{\Omega}^{\mu^{\prime}}+\tilde{\mu}_{m^{\prime} n^{\prime}} \tilde{\Sigma}^{m^{\prime} n^{\prime}} \\
& +\tilde{\mu}^{m}\left(\tilde{P}_{m}-\tilde{P}_{m^{\prime}}\right) \\
= & \frac{1}{2} \stackrel{\triangleright}{\underline{M}}_{\underline{M}}\left(\lambda_{\tau} \hat{\eta}^{\underline{M N}}+\lambda_{\sigma} \eta^{\underline{M N}}+\lambda \cdot \rho^{\underline{M N}}\right) \stackrel{\triangleright}{\triangleright}_{\underline{N}}+\mu^{\underline{M}}{\stackrel{\triangleright}{D_{M}}}_{\underline{\mu}}+\tilde{\mu}^{\underline{M}} \tilde{\triangleright}_{\underline{M}}
\end{aligned}
$$

with

$$
\begin{aligned}
& \mu^{\underline{M}}=\left(\mu^{S}, \mu^{S^{\prime}}, \text { others }=0\right) . \\
& \tilde{\mu}^{\underline{M}}=\left(\tilde{\mu}^{\Sigma}, \tilde{\mu}^{\Sigma^{\prime}}, \tilde{\mu}^{\Omega}, \tilde{\mu}^{\Omega^{\prime}}, \tilde{\mu}^{P}, \tilde{\mu}^{P^{\prime}}=-\tilde{\mu}^{P}, \text { others }=0\right) .
\end{aligned}
$$

The matrices $\rho \frac{M N}{}$ are nilpotent metrics introduced to represent $\mathcal{B C D}$ constraints [26]

$$
\left(\rho^{M N}\right)^{\mu} \stackrel{\circ}{\triangleright}_{M} \stackrel{\triangleright}{\triangleright}_{N}=\mathcal{B}^{\mu},\left(\rho^{M N}\right)_{\mu \nu} \stackrel{\triangleright}{\triangleright}_{M} \stackrel{\circ}{\triangleright}_{N}=\mathcal{C}_{\mu \nu},\left(\rho^{M N}\right)^{m} \stackrel{\triangleright}{\triangleright}_{M} \stackrel{\circ}{\triangleright}_{N}=\mathcal{D}^{m}
$$

and the similar relation for the right sector. An action for a type II superstring in the doubled space is given by

$$
I=\int d \tau d \sigma \mathcal{L}, \quad \mathcal{L}=\partial_{\tau} Z^{\underline{M}} \frac{1}{i} \partial_{\underline{M}}-\mathcal{H}=J_{0}^{\underline{M}} \stackrel{\circ}{\nabla}_{\underline{M}}-\mathcal{H}
$$

The analogous relations in (3.34) and (3.35) hold for a supersymmetric case. From the triangle property of the matrix $M_{I}^{J}$ they are rewritten as

$$
\begin{aligned}
\tilde{P} & =M_{P}{ }^{P}\left(P-J_{1}^{P}\right)+M_{P}{ }^{\Omega}\left(M^{-1}\right)_{\Omega}{ }^{\Omega} \tilde{\Omega}+\left(\left(M^{-1}\right)_{\Omega^{\Sigma}}{ }^{\Sigma} M_{P}{ }^{\Sigma}\left(M^{-1}\right)_{\Sigma}{ }^{\Sigma}\right) \tilde{\Sigma} \\
\tilde{P}^{\prime} & =M_{P^{\prime}} P^{\prime}\left(P^{\prime}+J_{1}^{P^{\prime}}\right)+M_{P^{\prime}} \Omega^{\prime}\left(M^{-1}\right)_{\Omega^{\prime}} \Omega^{\prime} \tilde{\Omega}^{\prime}+\left(\left(M^{-1}\right)_{\Omega^{\prime}} \Sigma^{\prime}+M_{P^{\prime}}{ }^{\Sigma^{\prime}}\left(M_{\Sigma^{\prime}}{ }^{\prime}\right)^{-1}\right) \tilde{\Sigma}^{\prime} \\
\stackrel{\circ}{\nabla}_{\Omega} & =\frac{1}{2}\left(J_{1}{ }^{D}-J_{1}{ }^{K} B_{K \Omega}\right)+\left(M^{-1}\right)_{\Omega^{\Omega}} \tilde{\Omega}+\left(M^{-1}\right)_{\Omega^{\Sigma}} \tilde{\Sigma} \\
\stackrel{\circ}{\nabla}_{\Sigma} & =\frac{1}{2}\left(J_{1}{ }^{S}-J_{1}{ }^{K} B_{K \Sigma}\right)+\left(M^{-1}\right)_{\Sigma}{ }^{\Sigma} \tilde{\Sigma} .
\end{aligned}
$$

Then the Lagrangian is rewritten as

$$
\begin{aligned}
& \mathcal{L}=\mathcal{L}_{0}+\mathcal{L}_{W Z}+\mathcal{L}_{W Z ; 0}+\mathcal{L}_{\text {const }} \\
& \left\{\begin{aligned}
\mathcal{L}_{0}= & J_{0}{ }^{P} P-\frac{\lambda}{2} P^{2}-\frac{1}{2} J_{0}{ }^{P} J_{1}{ }^{P}-\tilde{\mu}^{P}\left(M_{P} P\left(P-J_{1}{ }^{P}\right)-M_{P^{\prime}} P^{\prime}\left(P^{\prime}+J_{1} P^{\prime}\right)\right) \\
& +J_{0} P^{\prime} P^{\prime}-\frac{\lambda^{\prime}}{2} P^{\prime 2}+\frac{1}{2} J_{0} P^{\prime} J_{1} P^{\prime}+\rho D+\rho^{\prime} D^{\prime} \\
\mathcal{L}_{W Z}= & -\frac{1}{2}\left(J_{0}{ }^{N} J_{1}{ }^{M} B_{M N}-J_{0}{ }^{\prime} J_{1}{ }^{M^{\prime}} B_{M^{\prime} N^{\prime}}\right) \\
\mathcal{L}_{W Z ; 0}= & \frac{1}{2}\left(J_{[0}{ }^{\Sigma} J_{1]} S+J_{[0}{ }^{\Omega} J_{1]}{ }^{D}-J_{[0}{ }^{\Sigma^{\prime}} J_{1]}{ }^{S^{\prime}}-J_{[0}{ }^{\Omega^{\prime}} J_{1]}{ }^{\prime}\right) \\
\mathcal{L}_{\text {const }}= & \rho \cdot D+\rho^{\prime} \cdot D^{\prime}-\hat{\mu}^{S} S-\hat{\mu}^{S^{\prime}} S^{\prime}-\hat{\mu}^{\Omega} \tilde{\Omega}-\hat{\mu}^{\Omega^{\prime}} \tilde{\Omega}^{\prime}-\hat{\mu}^{\Sigma} \tilde{\Sigma}-\hat{\mu}^{\Sigma^{\prime}} \tilde{\Sigma}^{\prime}
\end{aligned}\right.
\end{aligned}
$$


by suitable redefinition of the Lagrange multipliers similar to (3.38) except $\rho$ 's

$$
\begin{aligned}
& \rho=J_{0}{ }^{D}-\lambda \Omega-\lambda_{\mu} \rho^{\mu} \not P-\lambda^{\mu \nu} \rho_{\mu \nu} D-\lambda_{m} \rho^{m} \partial_{\sigma} D \\
& \rho^{\prime}=J_{0} D^{\prime}-\lambda^{\prime} \Omega^{\prime}-\lambda_{\mu^{\prime}} \rho^{\mu^{\prime}} \not^{\prime}-\lambda^{\mu^{\prime} \nu^{\prime}} \rho_{\mu^{\prime} \nu^{\prime}} D^{\prime}-\lambda_{m^{\prime}} \rho^{m^{\prime}} \partial_{\sigma} D^{\prime}
\end{aligned}
$$

The first class constraints $\mathcal{B C D}$ are included in $\rho \cdot D$ and $\rho^{\prime} \cdot D^{\prime}$ terms in $\mathcal{L}_{\text {const }}$.

In order to compare it with the Green-Schwarz action we use the second class constraints $D_{\mu}=D_{\mu^{\prime}}=0$ instead of first class constraints $\mathcal{B C D}=0$. The kinetic term becomes the same as the bosonic one $\mathcal{L}_{0}$, while the Wess-Zumino term includes bilinears in the fermionic currents. We impose the fermionic second class constraints in addition to the first class constraints $D_{\mu}=D_{\mu^{\prime}}=0$. The resultant gauge invariant action for a type II superstring in the doubled space is given by

$$
\begin{gathered}
I=\int d \tau d \sigma \mathcal{L}, \quad \mathcal{L}=\mathcal{L}_{0}+\mathcal{L}_{W Z}+\mathcal{L}_{W Z ; 0} \\
\left\{\begin{array}{c}
\mathcal{L}_{0}=\frac{\alpha \beta}{2}\left[\sqrt{-h} h^{i j} J_{i}^{\mathcal{P}+} J_{j}^{\mathcal{P}+}-\epsilon^{i j} J_{i}^{\mathcal{P}+} J_{j}^{\mathcal{P}-}\right] \\
\mathcal{L}_{W Z}=-\frac{\alpha \beta}{2}\left(J_{0}{ }^{N} J_{1}{ }^{M} B_{M N}-J_{0}{ }^{N^{\prime}} J_{1}{ }^{M^{\prime}} B_{M^{\prime} N^{\prime}}\right) \\
\mathcal{L}_{W Z ; 0}=\frac{\alpha \beta}{2}\left(J_{[0}{ }^{\Sigma} J_{1]}{ }^{S}+J_{[0}{ }^{\Omega} J_{1]}{ }^{D}-J_{[0}{ }^{{ }^{\prime}} J_{1]}{ }^{S^{\prime}}-J_{[0} \Omega^{\prime} J_{1]}{ }^{D^{\prime}}\right)
\end{array}\right.
\end{gathered}
$$

where

$$
\begin{aligned}
J_{i}^{\mathcal{P} \pm} & =\left(J_{i}^{P} \pm J_{i}^{P^{\prime}} \underline{M}_{P^{\prime}}^{P}\right) / \sqrt{2} \\
\underline{M}_{P^{\prime}}{ }^{P} & =\left(M^{-1}\right)_{P^{\prime}} P^{\prime} M_{P}{ }^{P} .
\end{aligned}
$$

The normalization parameter $\alpha \beta$ given in (3.6) and (3.7) is natural to set $\alpha \beta=2$. The Lagrange multipliers $\rho$ and $\rho^{\prime}$ are

$$
\rho=J_{0}^{D}-\lambda \Omega, \quad \rho^{\prime}=J_{0}^{D^{\prime}}-\lambda^{\prime} \Omega^{\prime} .
$$

Taking variation of the action (4.14) with respect to the super-coordinates $Z \underline{M}$ the following first class constraints are derived; the fermionic second class and the $\kappa$-symmetry first class constraints, Virasoro constraints in (3.8), the isotropy constraints (3.22), dimensional reduction constraints (3.23) and the left/right mixing dimensional reduction constraint (3.24)

$$
\begin{aligned}
& \mathcal{H}_{\tau}=\mathcal{H}_{\sigma}=D_{\mu}=D_{\mu^{\prime}}=S_{m n}=S_{m^{\prime} n^{\prime}}=0 \\
& \tilde{\Omega}^{\mu}=\tilde{\Omega}^{\mu^{\prime}}=\tilde{\Sigma}^{m n}=\tilde{\Sigma}^{m^{\prime} n^{\prime}}=\tilde{P}_{m}-\tilde{P}_{m^{\prime}}=0 .
\end{aligned}
$$

Type IIA or IIB is determined by the gamma matrix chiral projection obtained from the algebra between supercharges $\tilde{D}_{\mu}$ and $\tilde{D}_{\mu^{\prime}}$ and the total Lorentz charge $\left(\tilde{S}-\tilde{S}^{\prime}\right)_{\mathrm{mn}}$.

Under the global $\mathrm{O}(\mathrm{n}, \mathrm{n})$ transformation, $S_{m n}$ and $\Sigma^{m n}$ components of the $\mathrm{O}(\mathrm{n}, \mathrm{n})$ matrix are treated as same as the bosonic case (3.30). Its fermionic components, $D_{\mu}$ and 
$\Omega^{\mu}$, involve the Ramond-Ramond gauge field dimensions $\Upsilon_{\mu \nu^{\prime}}$ and $F^{\mu \nu^{\prime}}$ introduced in [28]. This issue will be discussed in another paper.

In the simple solution for $B$ field in (2.25) the Lagrangian becomes

$$
\mathcal{L}=\sqrt{-h} h^{i j} J_{i}^{\mathcal{P}+} J_{j}^{\mathcal{P}+}+\epsilon^{i j}\left(-J_{i}^{\mathcal{P}+} J_{j}^{\mathcal{P}-}+\frac{1}{2} J_{i}^{\Omega} J_{j}{ }^{D}-\frac{1}{2} J_{i}^{\Omega^{\prime}} J_{j}^{D^{\prime}}\right)
$$

If further simple gauge $\underline{M}_{P^{\prime}}{ }^{P}=1$ and the constant $B$ field are used, then it reduces into the $(p, q)$-brane action proposed by Sakaguchi $[39,40]$ which is obtained from the central extended superalgebra. The manifest $\mathrm{SL}(2)$ S-duality is proposed into the $(p, q)$ brane action [41]. The manifest $S$ and T-duality action will be unified in the F-theory [42-44]. With the gauge fixing condition $J_{i}^{\mathcal{P}-}=0$ and ignoring the surface term, it reduces to the usual Green-Schwarz superstring action.

\section{Conclusions}

In this paper we have presented general construction of chiral affine Lie algebras generated by the supercovariant derivatives and the symmetry generators for a type II superstring. The covariant derivatives and the symmetry generators have the general form given in (2.26) where the $B$ field is determined from the relation (2.23) and (2.24). There is a constant solution of the $B$ field (2.25) where the nondegenerate group metric and the dilatation operator play essential roles.

The obtained covariant derivatives and symmetry generators become chiral by doubling the Lie group. Chirality is manifest in the doubled space; each coordinate is a function of only the left or right moving coordinate in a string worldsheet as $Z^{M}\left(\sigma^{+}\right)$and $Z^{M^{\prime}}\left(\sigma^{-}\right)$. Nondegeneracy of the group gives the unique chiral representations. The supercovariant derivatives are manifestly chiral even after the dimensional reduction into the usual space.

The doubled space is reduced into the usual space by a set of dimensional reduction constraints. Auxiliary directions introduced for the nondegeneracy of the group are reduced by using symmetry generators, since symmetry generators commute with covariant derivatives. So the local geometry governed by the covariant derivatives is preserved under the dimensional reduction. Therefore the local geometry of the doubled space with manifest T-duality is preserved.

Gauge invariant actions for a bosonic string and a type II superstring in the doubled space are obtained in (3.42) and (4.14) respectively. The resultant actions include the kinetic term, the Wess-Zumino term and the boundary of the Wess-Zumino term. There exists the winding mode contribution through the term $J^{\mathcal{P}+} \wedge J^{\mathcal{P}-}$, which can be gauged away by the constraint.

T-duality transformations on branes in the doubled space and the M-theory and the F-theory extension will be interesting issues.

\section{Acknowledgments}

W.S. thanks to Martin Poláček for valuable discussions. M.H. would like to thank the Simons Center for Geometry and Physics for hospitality during "the 2014 Summer Simons 
workshop in Mathematics and Physics" where this work has been developed. The work of M.H. is supported by Grant-in-Aid for Scientific Research (C) No. 24540284 from The Ministry of Education, Culture, Sports, Science and Technology of Japan, and the work of W.S. is supported in part by National Science Foundation Grant No. PHY-1316617.

\section{A Composition formula}

We first recall the composition formula valid for small $t$

$$
\begin{aligned}
e^{t A} e^{B} & =e^{B+t C_{+}}, \quad C_{+}=\sum_{n=0} b_{n}\left([B,-)^{n} A=\sum_{n=0} b_{n}\left(\operatorname{adj}_{B}\right)^{n} A,\right. \\
e^{B} e^{t A} & =e^{B+t C_{-}}, \quad C_{-}=\sum_{n=0}(-)^{n} b_{n}\left(\operatorname{adj}_{B}\right)^{n} A, \\
\sum_{r=0} b_{r} t^{r} & =\frac{t}{e^{t}-1}, \quad \sum_{r=\text { even }} b_{r} t^{r}=\frac{t}{2} \frac{e^{t}+1}{e^{t}-1}, \quad \sum_{r=o d d} b_{r} t^{r}=\frac{-t}{2}, \\
b_{0} & =1, \quad b_{1}=-\frac{1}{2}, \quad b_{2}=\frac{1}{12}, \quad b_{3}=0, \quad b_{4}=-\frac{1}{720}, \ldots
\end{aligned}
$$

where in the first line $\left([B,-)^{n} A=\left(\operatorname{adj}_{B}\right)^{n} A\right.$ means the $\mathrm{n}$ times commutation operations between $B$, as $[B,[B, \ldots[B, A] \ldots]]$. Consider a operator relation for small $t$

$$
e^{t A} e^{B}=e^{C}, \quad C=C_{0}+t C_{1}+\frac{t^{2}}{2} C_{2}+\ldots
$$

At $t=0 \quad C_{0}=B$. By taking $t$ derivative

$$
e^{t A} A e^{B}=\int_{0}^{1} d s e^{s C}\left(C_{1}+t C_{2}+\ldots\right) e^{(1-s) C}
$$

and put $t=0$

$$
\begin{aligned}
A & =\int_{0}^{1} d s e^{s B} C_{1} e^{-s B}=\int_{0}^{1} d s\left(C_{1}+s\left[B, C_{1}\right]+\frac{s^{2}}{2 !}\left[B,\left[B, C_{1}\right]\right]+\ldots\right) \\
& =C_{1}+\frac{1}{2}\left[B, C_{1}\right]+\frac{1}{3 !}\left[B,\left[B, C_{1}\right]\right]+\ldots=\sum_{n=0} \frac{1}{(n+1) !}\left(\operatorname{adj}_{B}\right)^{n} C_{1} .
\end{aligned}
$$

The solution $C_{1}$ is found in the form

$$
C_{1}=\sum_{n=0} b_{n}\left(\operatorname{adj}_{B}\right)^{n} A .
$$

Put it back into (A.5)

$$
A=\sum_{n=0} \frac{1}{(n+1) !}\left(\operatorname{adj}_{B}\right)^{n} \sum_{k=0} b_{k}\left(\operatorname{adj}_{B}\right)^{k} A=\sum_{n=0} \sum_{k=0}^{n} \frac{b_{k}}{(n-k+1) !}\left(\operatorname{adj}_{B}\right)^{n} A
$$

From here we find

$$
b_{0}=1, \quad \text { and } \quad b_{r}=-\sum_{k=0}^{r-1} \frac{b_{k}}{(r-k+1) !},(r=1,2, \ldots) .
$$


The latter is solved recursively.

$$
b_{0}=1, \quad b_{1}=-\frac{1}{2}, \quad b_{2}=\frac{1}{12}, \quad b_{3}=0, \quad b_{4}=-\frac{1}{720}, \ldots
$$

We make a generating function

$$
\begin{aligned}
G(t) & =\sum_{r=0} b_{r} t^{r}=b_{0}-\sum_{r=1} t^{r} \sum_{k=0}^{r-1} \frac{b_{k}}{(r-k+1) !}=1-\sum_{k=0}^{\infty} \sum_{r=k+1}^{\infty} \frac{t^{r} b_{k}}{(r-k+1) !} \\
& =1-\sum_{k=0}^{\infty} \sum_{s=0}^{\infty} \frac{t^{s+k+1} b_{k}}{(s+2) !}=1-\sum_{s=0}^{\infty} \frac{t^{s+1}}{(s+2) !} G(t) .
\end{aligned}
$$

From this we can solve $G(t)$ as

$$
G(t)=\frac{t}{e^{t}-1}=\frac{1}{1+\frac{t}{2 !}+\frac{t^{2}}{3 !}+\ldots}
$$

The coefficients $b_{n}$ is found by

$$
b_{n}=\lim _{t \rightarrow 0} \frac{1}{n !}\left(\partial_{t}\right)^{n} G(t) .
$$

Thus the solution of (A.3) is

$$
C_{1}=\sum_{n=0} b_{n}\left(\operatorname{adj}_{B}\right)^{n} A=G\left(\operatorname{adj}_{B}\right) A=\frac{\left(\operatorname{adj}_{B}\right)}{e^{\left(\operatorname{adj}_{B}\right)}-1} A
$$

$b_{n}$ is related to the Bernoulli numbers $B_{n}$ by

$$
b_{n}=\frac{B_{n}}{n !} .
$$

More generally the Bernoulli function $B_{n}(x)$ is defined by

$$
G(t, x)=\frac{t e^{t x}}{e^{t}-1}=\sum_{n=0}^{\infty} \frac{t^{n}}{n !} B_{n}(x) .
$$

\section{B Nonlinear realization of nondegenerate Poincaré groups}

The left-invariant one form for a Lorentz group is calculated as follows. Using an abbreviated notation $u \cdot s=\frac{1}{2} u^{m n} s_{m n}$, the canonical dimension zero part is given $g^{-1} d g=$ $e^{-i u \cdot s} d e^{i u \cdot s}+e^{-i u^{\prime} \cdot s^{\prime}} d e^{i u^{\prime} \cdot s^{\prime}}$

$$
\begin{aligned}
e^{-i u \cdot s} d e^{i u \cdot s} & =\int_{0}^{1} d t e^{-i t u \cdot s} i d u \cdot s e^{i t u \cdot s} \\
& =i d u \cdot s+\frac{1}{2}[-i u \cdot s, i d u \cdot s]+\frac{1}{3 !}[-i u \cdot s[-i u \cdot s, i d u \cdot s]]+\cdots \\
& =\frac{e^{\operatorname{adj}_{u}}-1}{\operatorname{adj}_{u}} i d u \cdot s \equiv \Xi_{u}^{-1} i d u \cdot s
\end{aligned}
$$


$\Xi^{-1}$ is an inverse of $\Xi$ which is given by

$$
\Xi_{u}=\frac{\operatorname{adj}_{u}}{e^{\operatorname{adj}_{u}-1}}, \operatorname{adj}_{u} A=[u, A]
$$

expressed by the Bernoulli number $B_{n}$ as

$$
\begin{aligned}
\frac{q}{e^{q}-1} & =\sum_{n=0} B_{n} \frac{q^{n}}{n !} \\
B_{0}=1, B_{1}=-\frac{1}{2}, B_{2}=\frac{1}{6}, B_{3} & =0, B_{4}=-\frac{1}{30}, \cdots, B_{2 m+1}=0(m \neq 0) \\
\sum_{n=\text { even }} B_{n} \frac{q^{n}}{n !} & =\frac{q}{2} \frac{e^{q}+1}{e^{q}-1}, \quad \sum_{n=\text { odd }} B_{n} \frac{q^{n}}{n !}=-\frac{q}{2} .
\end{aligned}
$$

The resultant left-invariant current and right-invariant currents of doubled nondegenerate Poincaré algebras are followings:

- Left-invariant currents

$$
\begin{aligned}
& \text { Flat left } J^{M}=\left(J^{S m n}, J^{P m}, J^{\Sigma_{m n}}\right) \\
& \left\{\begin{array}{l}
J^{S m n}=\left(e^{-u}\right)^{m l} d\left(e^{u}\right)^{l n}=\left(\Xi_{u}^{-1}\right)^{m l} d u^{l n} \\
J^{P m}=\left(e^{-u}\right)^{m n} d x^{n} \\
J^{\Sigma_{m n}}=\left(e^{-u}\right)_{m l}\left(d v_{l k}+\frac{1}{2} x_{[l} d x_{k]}\right)\left(e^{u}\right)_{k n}
\end{array}\right.
\end{aligned}
$$

$$
\begin{aligned}
& \text { Flat right } J^{M^{\prime}}=\left(J^{S^{\prime} m^{\prime} n^{\prime}}, J^{P^{\prime} m^{\prime}}, J^{\Sigma^{\prime}}{ }_{m^{\prime} n^{\prime}}\right) \\
& \left\{\begin{array}{l}
J^{S^{\prime} m^{\prime} n^{\prime}}=-\left(e^{u^{\prime}}\right)^{m^{\prime} l^{\prime}} d\left(e^{-u^{\prime}}\right)^{l^{\prime} n^{\prime}} \\
J^{P^{\prime} m^{\prime}}=\left(e^{u^{\prime}}\right)^{m^{\prime} n^{\prime}} d x^{\prime n^{\prime}} \\
J^{\Sigma^{\prime}{ }_{m^{\prime} n^{\prime}}}=\left(e^{u^{\prime}}\right)^{m^{\prime} l^{\prime}}\left(d v^{\prime} l^{\prime} k^{\prime}-\frac{1}{2} x^{\prime}{ }_{l^{\prime}}, d x^{\prime}{ }_{k^{\prime}}\right]\left(e^{-u^{\prime}}\right)_{k^{\prime} n^{\prime}}
\end{array}\right.
\end{aligned}
$$

- Right-invariant currents

Flat left $\tilde{J}^{M}=\left(\tilde{J}^{S m n}, \tilde{J}^{P m}, \tilde{J}^{\Sigma}{ }_{m n}\right)$

$$
\left\{\begin{aligned}
\tilde{J}^{S m n} & =-\left(e^{u}\right)^{m l} d\left(e^{-u}\right)^{l n} \\
\tilde{J}^{P m} & =d x^{m}-\tilde{J}^{S m n} x^{n} \\
\tilde{J}^{\Sigma}{ }_{m n} & =d v_{m n}-\frac{1}{2} x_{[m} \tilde{J}_{n]}^{P}+v_{[m \mid l} \tilde{J}_{l \mid n]}
\end{aligned}\right.
$$

Flat right $\tilde{J}^{M^{\prime}}=\left(\tilde{J}^{S^{\prime} m^{\prime} n^{\prime}}, \tilde{J}^{P^{\prime} m^{\prime}}, \tilde{J}^{\Sigma^{\prime}{ }_{m^{\prime} n^{\prime}}}\right)$

$$
\left\{\begin{aligned}
\tilde{J}^{S^{\prime} m^{\prime} n^{\prime}} & =\left(e^{-u^{\prime}}\right)^{m^{\prime} l^{\prime}} d\left(e^{u^{\prime}}\right)^{l^{\prime} n^{\prime}} \\
\tilde{J}^{P^{\prime} m^{\prime}} & =d x^{\prime m^{\prime}}+\tilde{J}^{S^{\prime} m^{\prime} n^{\prime}} x^{\prime n^{\prime}} \\
\tilde{J}^{\Sigma^{\prime}}{ }_{m^{\prime} n^{\prime}} & =d v^{\prime}{ }_{m^{\prime} n^{\prime}}+\frac{1}{2} x^{\prime}{ }_{\left[m^{\prime} \mid l^{\prime}\right.} \tilde{J}^{P^{\prime}}{ }_{\left.l^{\prime} \mid n^{\prime}\right]}+v_{\left[m^{\prime} \mid l^{\prime}\right.} \tilde{J}^{S^{\prime}}{ }_{\left.l^{\prime} \mid n^{\prime}\right]}
\end{aligned}\right.
$$


They satisfy the following Maurer-Cartan equations.

- The Maurer-Cartan for the left-invariant currents

Flat left

$$
\left\{\begin{array}{l}
d J^{S}+J^{S} \wedge J^{S}=0 \\
d J^{P}+J^{S} \wedge J^{P}=0 \\
d J^{\Sigma}+J^{S} \wedge J^{\Sigma}+J^{\Sigma} \wedge J^{S}-J^{P} \wedge J^{P}=0
\end{array}\right.
$$

Flat right

$$
\left\{\begin{array}{l}
d J^{S^{\prime}}-J^{S^{\prime}} \wedge J^{S^{\prime}}=0 \\
d J^{P^{\prime}}-J^{S^{\prime}} \wedge J^{P^{\prime}}=0 \\
d J^{\Sigma^{\prime}}-J^{S^{\prime}} \wedge J^{\Sigma^{\prime}}-J^{\Sigma^{\prime}} \wedge J^{S^{\prime}}+J^{P^{\prime}} \wedge J^{P^{\prime}}=0
\end{array}\right.
$$

- The Maurer-Cartan equations for the right-invariant currents

$$
\begin{aligned}
& \text { Flat left } \\
& \left\{\begin{array}{l}
d \tilde{J}^{S}-\tilde{J}^{S} \wedge \tilde{J}^{S}=0 \\
d \tilde{J}^{P}-\tilde{J}^{S} \wedge \tilde{J}^{P}=0 \\
d \tilde{J}^{\Sigma}-\tilde{J}^{S} \wedge \tilde{J}^{\Sigma}-\tilde{J}^{\Sigma} \wedge \tilde{J}^{S}+\tilde{J}^{P} \wedge \tilde{J}^{P}=0
\end{array}\right.
\end{aligned}
$$

Flat right

$$
\left\{\begin{array}{l}
d \tilde{J}^{S^{\prime}}+\tilde{J} S^{\prime} \wedge \tilde{J} S^{\prime}=0 \\
d \tilde{J}^{P^{\prime}}+\tilde{J} S^{S^{\prime}} \wedge \tilde{J}^{P^{\prime}}=0 \\
d \tilde{J}^{\Sigma^{\prime}}+\tilde{J} S^{S^{\prime}} \wedge \tilde{J}^{\Sigma^{\prime}}+\tilde{J}^{\Sigma^{\prime}} \wedge \tilde{J}^{S^{\prime}}-\tilde{J}^{P^{\prime}} \wedge \tilde{J}^{P^{\prime}}=0
\end{array}\right.
$$

where Lorentz indices are contracted with nearest neighbor indices.

Open Access. This article is distributed under the terms of the Creative Commons Attribution License (CC-BY 4.0), which permits any use, distribution and reproduction in any medium, provided the original author(s) and source are credited.

\section{References}

[1] W. Siegel, Covariant approach to superstrings, in Symposium on anomalies, geometry, topology, Chicago U.S.A. (1985), W.A. Bardeen and A.R. White eds., World Scientific, Singapore (1985), pg. 348. 
[2] W. Siegel, Covariant superstrings, in Unified string theories, Santa Barbara U.S.A. (1985), M. Green and D. Gross eds., World Scientific, Singapore (1985), pg. 559.

[3] W. Siegel, Classical Superstring Mechanics, Nucl. Phys. B 263 (1986) 93 [InSPIRE].

[4] W. Siegel, Randomizing the superstring, Phys. Rev. D 50 (1994) 2799 [hep-th/9403144] [INSPIRE].

[5] M.J. Duff, Duality Rotations in String Theory, Nucl. Phys. B 335 (1990) 610 [InSPIRE].

[6] A.A. Tseytlin, Duality Symmetric Formulation of String World Sheet Dynamics, Phys. Lett. B 242 (1990) 163 [INSPIRE].

[7] A.A. Tseytlin, Duality symmetric closed string theory and interacting chiral scalars, Nucl. Phys. B 350 (1991) 395 [InSPIRE].

[8] J. Maharana and J.H. Schwarz, Noncompact symmetries in string theory, Nucl. Phys. B 390 (1993) 3 [hep-th/9207016] [INSPIRE].

[9] W. Siegel, Two vierbein formalism for string inspired axionic gravity, Phys. Rev. D 47 (1993) 5453 [hep-th/9302036] [INSPIRE].

[10] W. Siegel, Superspace duality in low-energy superstrings, Phys. Rev. D 48 (1993) 2826 [hep-th/9305073] [INSPIRE].

[11] W. Siegel, Manifest duality in low-energy superstrings, hep-th/9308133 [INSPIRE].

[12] N. Hitchin, Generalized Calabi-Yau manifolds, Quart. J. Math. 54 (2003) 281 [math/0209099] [INSPIRE].

[13] M. Gualtieri, Generalized complex geometry, math/0401221.

[14] C.M. Hull, A Geometry for non-geometric string backgrounds, JHEP 10 (2005) 065 [hep-th/0406102] [INSPIRE].

[15] C.M. Hull, Doubled Geometry and T-Folds, JHEP 07 (2007) 080 [hep-th/0605149] [INSPIRE].

[16] C.M. Hull, Generalised Geometry for M-theory, JHEP 07 (2007) 079 [hep-th/0701203] [INSPIRE].

[17] M. Graña, Flux compactifications in string theory: A Comprehensive review, Phys. Rept. 423 (2006) 91 [hep-th/0509003] [INSPIRE].

[18] C. Hull and B. Zwiebach, Double Field Theory, JHEP 09 (2009) 099 [arXiv:0904.4664] [INSPIRE].

[19] O. Hohm, C. Hull and B. Zwiebach, Generalized metric formulation of double field theory, JHEP 08 (2010) 008 [arXiv: 1006.4823] [INSPIRE].

[20] O. Hohm, S.K. Kwak and B. Zwiebach, Unification of Type II Strings and T-duality, Phys. Rev. Lett. 107 (2011) 171603 [arXiv:1106.5452] [INSPIRE].

[21] I. Jeon, K. Lee and J.-H. Park, Ramond-Ramond Cohomology and O(D,D) T-duality, JHEP 09 (2012) 079 [arXiv:1206.3478] [INSPIRE].

[22] I. Jeon, K. Lee, J.-H. Park and Y. Suh, Stringy Unification of Type IIA and IIB Supergravities under $N=2 D=10$ Supersymmetric Double Field Theory, Phys. Lett. B 723 (2013) 245 [arXiv:1210.5078] [INSPIRE].

[23] R. Blumenhagen, F. Hassler and D. Lüst, Double Field Theory on Group Manifolds, JHEP 02 (2015) 001 [arXiv: 1410.6374] [INSPIRE]. 
[24] G. Aldazabal, D. Marques and C. Núñez, Double Field Theory: A Pedagogical Review, Class. Quant. Grav. 30 (2013) 163001 [arXiv:1305.1907] [INSPIRE].

[25] O. Hohm, D. Lüst and B. Zwiebach, The Spacetime of Double Field Theory: Review, Remarks and Outlook, Fortsch. Phys. 61 (2013) 926 [arXiv:1309.2977] [INSPIRE].

[26] M. Hatsuda, K. Kamimura and W. Siegel, Superspace with manifest T-duality from type-II superstring, JHEP 06 (2014) 039 [arXiv: 1403.3887] [INSPIRE].

[27] M. Poláček and W. Siegel, T-duality off shell in 3D Type II superspace, JHEP 06 (2014) 107 [arXiv: 1403.6904] [INSPIRE].

[28] M. Hatsuda, K. Kamimura and W. Siegel, Ramond-Ramond gauge fields in superspace with manifest T-duality, JHEP 02 (2015) 134 [arXiv:1411.2206] [INSPIRE].

[29] E. Witten, Nonabelian Bosonization in Two-Dimensions, Commun. Math. Phys. 92 (1984) 455 [inSPIRE].

[30] M. Hatsuda and K. Kamimura, Classical AdS superstring mechanics, Nucl. Phys. B 611 (2001) 77 [hep-th/0106202] [INSPIRE].

[31] M. Hatsuda, Sugawara form for AdS superstring, Nucl. Phys. B 730 (2005) 364 [hep-th/0507047] [INSPIRE].

[32] W. Siegel, Manifest Lorentz Invariance Sometimes Requires Nonlinearity, Nucl. Phys. B 238 (1984) 307 [inSPIRE].

[33] E. Cremmer, B. Julia, H. Lü and C.N. Pope, Dualization of dualities. 2. Twisted self-duality of doubled fields and superdualities, Nucl. Phys. B 535 (1998) 242 [hep-th/9806106] [INSPIRE].

[34] S.F. Hassan, $\mathrm{SO}(d, d)$ transformations of Ramond-Ramond fields and space-time spinors, Nucl. Phys. B 583 (2000) 431 [hep-th/9912236] [INSPIRE].

[35] W. Siegel, New superspaces/algebras for superparticles/strings, arXiv:1106.1585 [INSPIRE].

[36] M. Poláček and W. Siegel, Natural curvature for manifest T-duality, JHEP 01 (2014) 026 [arXiv: 1308.6350] [INSPIRE].

[37] S. Bonanos, J. Gomis, K. Kamimura and J. Lukierski, Maxwell Superalgebra and Superparticle in Constant Gauge Badkgrounds, Phys. Rev. Lett. 104 (2010) 090401 [arXiv: 0911.5072] [INSPIRE].

[38] M. Hatsuda and T. Kimura, Canonical approach to Courant brackets for D-branes, JHEP 06 (2012) 034 [arXiv:1203.5499] [INSPIRE].

[39] M. Sakaguchi, Type II superstrings and new space-time superalgebras, Phys. Rev. D 59 (1999) 046007 [hep-th/9809113] [INSPIRE].

[40] M. Sakaguchi, $(p, q)$-strings and new space-time superalgebras, JHEP 02 (1999) 017 [hep-th/9811143] [INSPIRE].

[41] M. Abe, M. Hatsuda, K. Kamimura and T. Tokunaga, $\mathrm{SO}(2,1)$ covariant IIB superalgebra, Nucl. Phys. B 553 (1999) 305 [hep-th/9903234] [INSPIRE].

[42] W.D. Linch and W. Siegel, F-theory Superspace, arXiv:1501.02761 [INSPIRE].

[43] W.D. Linch, III and W. Siegel, F-theory from Fundamental Five-branes, arXiv:1502.00510 [INSPIRE].

[44] W.D. Linch and W. Siegel, F-theory with Worldvolume Sectioning, arXiv:1503.00940 [INSPIRE]. 